\title{
VENTRICULAR CHANGES AFTER CLOSED HEAD INJURY
}

\author{
BY
}

HUGH DAVIES, Major R.A.M.C., T.A. and MURRAY A. FALCONER, T/Major, R.A.M.C.

(From a Military Hospital for Head Injuries)

(RECEIVED 7TH MAY, 1943)

\begin{abstract}
Introduction
Although the majority of patients who sustain a closed head injury appear to make a complete and satisfactory recovery, some are left with residual disabilities, which interfere with their well-being and their adjustment to society. It is known that in such cases permanent structural changes often occur in the brain, but clinically it is difficult to ascertain their severity and extent. There is no single method of investigation which affords this information. Such methods as neurological examination, radiological investigation of the skull, psychological tests, and electro-encephalography, each contribute something to the analysis, but each has its limitations. The following study was undertaken in order to evaluate air encephalography, not so much as a method of demonstrating the presence of posttraumatic space-occupying lesions, but of assessing the degree of damage which has occurred in the brain.

That changes in the outline of the ventricles often follow closed head injury was clearly established in papers from Foerster's clinic at Breslau by Schwab (1926a and $b$ ) and by Bielschowsky (1928). Both authors employed encephalography with air introduced by the lumbar route and both described three groups of cases with abnormal encephalographic findings, viz. (a) cases in which the ventricles did not fill; (b) cases with enlargement and deformity of the ventricles; and (c) cases with abnormalities of distribution of the sub-arachnoid air over the cerebral convexities. In addition both studied the circulation of the cerebro-spinal fluid after head injury by injecting sodium iodide solution into the ventricles or lumbar theca, and subsequently ascertaining the time of its appearance in other parts of the cerebro-spinal fluid system or in the urine. On comparing their findings with standards obtained from control cases, both of them concluded that in the majority of cases with abnormal encephalograms there were disturbances of the cerebro-spinal fluid circulation, either in the form of obstruction to the cerebro-spinal fluid pathways or of delayed absorption of the fluid into the bood stream. Bielschowsky, whose paper was based on 106 cases of head injury, of both closed and open types, went further than Schwab and tried to correlate the clinical features of his cases with the encephalographic appearances. He showed that, while in many citses there was an obvious relationship
\end{abstract}

between the two, in other cases this relationship was not apparent; and indeed the same encephalographic appearances as were seen in some cases with persistent symptoms and signs would also be seen in others without persistent symptoms or with only a history of post-traumatic epilepsy.

Since then several other authors have confirmed that encephalographic changes often follow head injury, but without exception they have based their findings on smaller groups of cases and have not extended the scope of Bielschowsky's observations. Fischer (1927), after investigating a series of 14 psychopathic patients, many of whom had sustained head injury several years previously, concluded that similar encephalographic abnormalities could sometimes be found in psychopathic patients who had no history of head injury, and that the correlation between clinical features and encephalographic findings was not always apparent. Swift (1931), Hauptmann (1932), Friedman (1932) and others have regarded encephalography as a means of differentiating between cases with genuine disabilities and cases with functional disturbances, implying that those cases which have normal encephalographic appearances usually belong to the latter group. Several others (Flugel 1932, Bennett and Hunt 1933, Lippens and Desjardin 1934, Travers 1937, Money and Susman 1932), have reported on the encephalographic changes following head injury without offering much comment on their ætiological and clinical significance.

There has thus been little advance in our appreciation of the encephalographic changes following head injury since Bielschowsky's paper appeared in 1928. During the intervening years knowledge of the clinical aspects of head injury has much improved. In this paper an attempt is therefore made to reassess, in terms of present-day clinical standards, the value of air encephalography in the investigation of cases of head injury after the acute stage.

\section{Case Material}

Our study is based upon 100 cases of closed head injury, which were admitted to a military hospital for head injuries, either as acute cases or for the investigation of symptoms deemed to be the result of injury. In all of these cases it was considered clinically that the symptoms did, in fact, result from the head injury. Cases with symptoms judged to be 
psychoneurotic in origin, or with symptoms considered to be referable to a previous mental or physical defect, were excluded. The series includes cases with depressed fractures, but not those with penetrating injuries. Together they form an unselected consecutive series of cases in which the full hospital notes and X-ray films were available and in which the encephalograms were satisfactory.

\section{Technique}

The cisternal route of injection was employed in all except 7 cases. The cerebro-spinal fluid was replaced fractionally with air, and the technique was directed to obtainir.g an adequate ventricular filling, but not a complete drainage of cerebro-spinal fluid, and thus a full outline of the sub-arachnoid spaces. The amount of air injected was usually between 30 and 60 c.c. This method was generally found adequate to demonstrate the ventricular changes without causing much discomfort to the patient, and without the necessity for employing a general anæsthetic. Its relative simplicity allowed of repeated examination in some cases.

Radiological examination with the six standard Lysholm (1935) projections was carried out, producing a composite picture of the entire ventricular system. The anode-film distance was kept constant at 36 inches; the $\mathrm{X}$-ray film was placed below the skull, and a fixed Lysholm grid was used in order to keep the head-film distance as low as possible and so reduce the amount of distortion, which would have resulted from the use of a PotterBucky diaphragm. Thus the radiological technique was standardized to permit of comparative measurements of the ventricles. It did not, however, permit of detailed studies of the distribution of air in the cortical subarachnoid spaces, such as would have been possible with lumbar encephalography and full drainage.

\section{Methods of Measurement}

Methods of measuring the ventricular system after encephalography have been described by Abramovitch and Winkler (1930), Davidoff and Dyke (1937), and Evans (1942). Abramovitch and Winkler recorded the ventricular measurements with a measuring stereoscope and related the ventricular to the cranial dimensions. Davidoff and Dyke took as their standards the maximum breadth and the length of the ventricular system in 150 cases considered to be normal. Evans compared in children the total breadth of the ventricular system with the

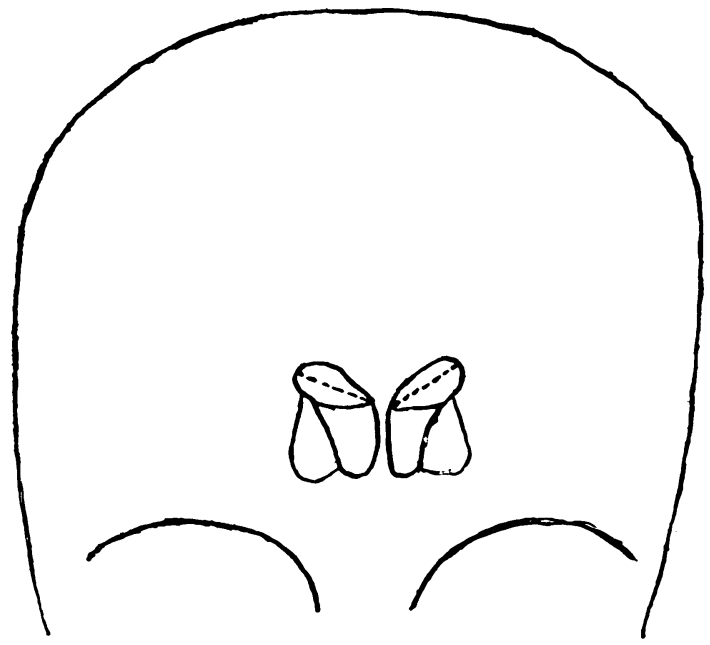

FIG. 1.-Outline of the lateral ventricles as projected in the brow-up antero-posterior view. The dotted line indicates the measurement taken for the assessment of ventricular size. maximum breadth of the skull. All these authors directed their measurements of the ventricles to the problem of establishing whether or not hydrocephalus was present.

Our problem was to assess not only general changes in one or both lateral ventricles but also focal changes. The latter occur most frequently in the frontal and temporal horns or in the trigone. From a study of our material it became apparent that the diagonal width of the body of the lateral ventricle formed an arbitrary but reliable criterion of the size of the lateral ventricle as a whole (Fig. 1). This measurement, which is most easily made in the standard brow-up projection, enables the size of the two lateral ventricles to be compared on the same film. Any variations due to inequality in filling of the two ventricles can be excluded by examination of the lateral projection with the brow up, in which view the amount of air in each lateral ventricle can be verified. Focal dilatation can be assessed by comparing component parts of the lateral ventricles with each other. This method of measurement was found to be reliable. In several cases repeated examination showed no change in the measurements obtained, thereby checking their accuracy, and also proving that repeated encephalography does not of itself lead to permanent ventricular changes.

As it had been suggested that variations in intracranial pressure, consequent upon the fractional replacement of cerebrospinal fluid, might cause temporary ventricular distension and so affect the measurements, some experiments were undertaken both on the cadaver and the living subject.

In recently dead cadavers injection of air directly into the ventricles under pressure was found to produce no change in the measurements of more than $1 \mathrm{~mm}$. In four cases, where encephalography was well tolerated, the quantity of air injected was deliberately increased relative to the quantity of fluid withdrawn. In a typical case films were obtained after the injection of 30 c.c. of air with equivalent withdrawal of fluid. A further 25 c.c. of air was then injected, at which point the patient complained of increasing severity of headache. A film was immediately obtained and cerebro-spinal fluid withdrawn to balance the replacement. No increase in the ventricular measurem.ents was observed (Fig. 2), either immediately or during the subsequent radiological procedure, extending over a period of nearly one hour. No alteration in m.easurements was noted in the other three cases similarly investigated (Table I). It is, therefore, suggested that the possibility of unreliable ventricular measurements, due to pressure changes arising during the injection of air, may be ignored.

Table I.-Ventricular Measurements in ConTROL CASES AFTER INTRODUCTION OF ADDITIONAL AIR

\begin{tabular}{|c|c|c|c|c|c|c|}
\hline & $\begin{array}{c}\text { Air } \\
\text { injected } \\
\text { with } \\
\text { equivalent } \\
\text { C.S.F. } \\
\text { with- } \\
\text { drawal } \\
\text { (c.c.) }\end{array}$ & $\begin{array}{l}\text { R.L.V. } \\
\text { (cm.) }\end{array}$ & $\begin{array}{l}\text { L.L.V. } \\
\text { (cm.) }\end{array}$ & $\begin{array}{l}\text { Air } \\
\text { injected } \\
\text { without } \\
\text { further } \\
\text { C.S.F. } \\
\text { with- } \\
\text { drawal } \\
\text { (c.c.) }\end{array}$ & $\begin{array}{l}\text { R.L.V. } \\
\text { (cm.) }\end{array}$ & $\begin{array}{l}\text { L.L.V. } \\
\text { (cm.) }\end{array}$ \\
\hline $\begin{array}{ll}\text { Case } 1 & \ldots \\
\text { Case } 2 & \ldots \\
\text { Case } 3 & \ldots \\
\text { Case } 4 & \ldots\end{array}$ & $\begin{array}{l}20 \\
30 \\
40 \\
35\end{array}$ & $\begin{array}{l}1.8 \\
1.7 \\
1.9 \\
1.8\end{array}$ & $\begin{array}{l}1.8 \\
1.8 \\
1.8 \\
1.7\end{array}$ & $\begin{array}{l}10 \\
25 \\
25 \\
20\end{array}$ & $\begin{array}{l}1.8 \\
1.7 \\
1.9 \\
1.8\end{array}$ & $\begin{array}{l}1.8 \\
1.8 \\
1.9 \\
1.7\end{array}$ \\
\hline
\end{tabular}

\section{Controls}

In order to establish standards for the normal ventricular measurements, we took a series of 50 cases (Table II), in which encephalography was considered desirable to exclude the presence of a space-occupying lesion, or which were not thought clinically to be examples of diseases usually associated with ventricular enlargement. In all of them the ventricular measurements were made under the same radiographic conditions as those in the head injury series, and in all of them the outlines 


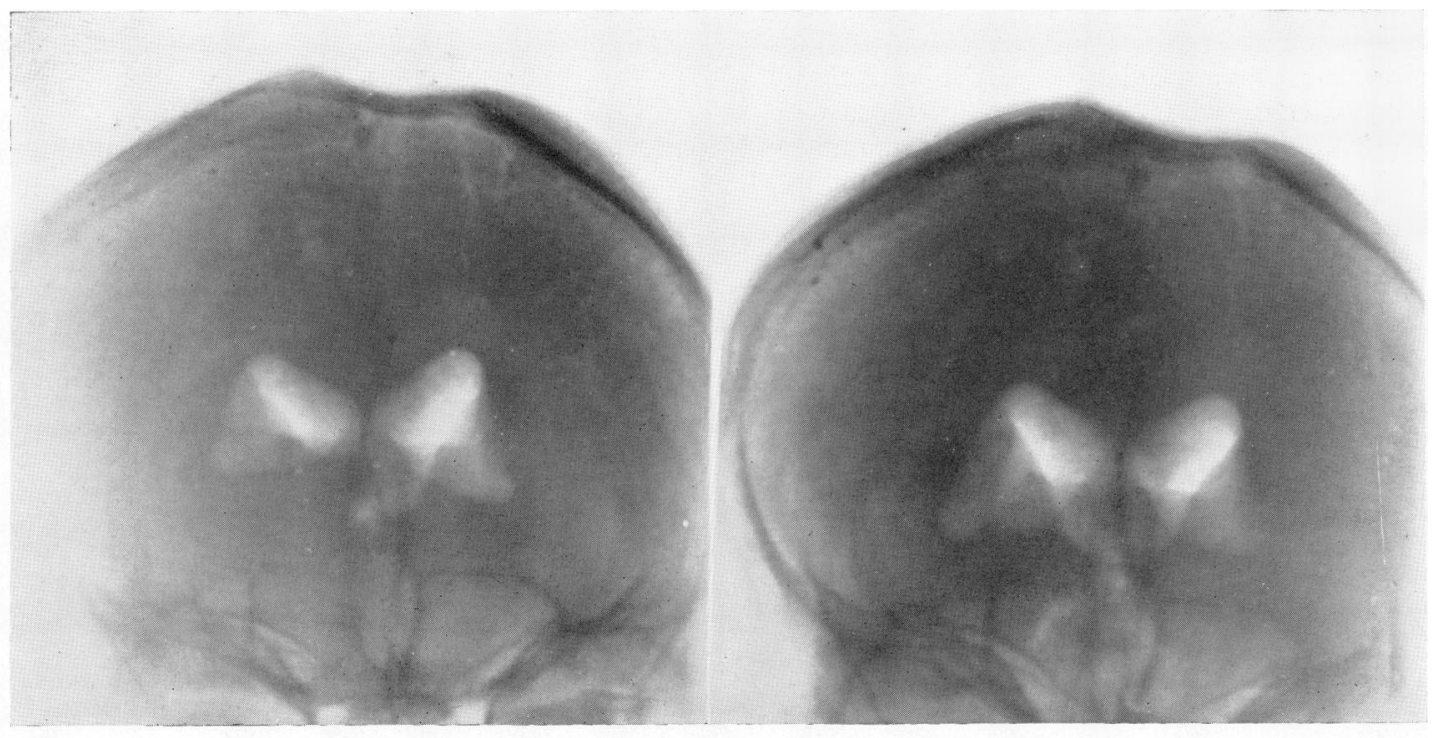

A

B

Fig. 2.-Table I. Case 3. A. Outline of ventricles after injection of 40 c.c. of air with equivalent withdrawal of c.s.f. B. Outline after the further injection of 25 c.c. of air without any withdrawal of c.s.f.

TABle II.-CONTROL Series

\begin{tabular}{|c|c|c|c|}
\hline $\begin{array}{l}\text { Hospital } \\
\text { case no. }\end{array}$ & Clinical diagnosis & $\begin{array}{l}\text { Ventr } \\
\text { measur } \\
\text { R.L.V. } \\
\text { (cm.) }\end{array}$ & $\begin{array}{l}\text { icular } \\
\text { ements } \\
\text { L.L.V. } \\
(\mathrm{cm} .)\end{array}$ \\
\hline 722 & Idiopathic epilepsy & $1 \cdot 5$ & $1 \cdot 5$ \\
\hline 881 & Idiopathic epilepsy & $1 \cdot 6$ & 1.6 \\
\hline 1498 & Idiopathic epilepsy & $1 \cdot 6$ & $1 \cdot 6$ \\
\hline 1775 & Idiopathic epilepsy & $1 \cdot 6$ & $1 \cdot 6$ \\
\hline$* 4209$ & Idiopathic encephalopathy & $1 \cdot 6$ & $1 \cdot 6$ \\
\hline 1332 & Migraine & $1 \cdot 6$ & $1 \cdot 6$ \\
\hline 401 & Psychoneurosis & $1 \cdot 6$ & $1 \cdot 6$ \\
\hline 938 & Familial optic atrophy & $1 \cdot 6$ & $1 \cdot 7$ \\
\hline 1396 & Idiopathic epilepsy & 1.6 & $1 \cdot 8$ \\
\hline 531 & Idiopathic epilepsy & $1 \cdot 7$ & $1 \cdot 6$ \\
\hline 3305 & Idiopathic epilepsy & $1 \cdot 7$ & $1 \cdot 7$ \\
\hline 1251 & Migraine & $1 \cdot 7$ & $1 \cdot 7$ \\
\hline 905 & Idiopathic epilepsy & $1 \cdot 7$ & $1 \cdot 8$ \\
\hline 3459 & Idiopathic epilepsy & $1 \cdot 7$ & $1 \cdot 8$ \\
\hline 1167 & Disseminated sclerosis & $1 \cdot 8$ & $1 \cdot 7$ \\
\hline 4853 & Migraine & $1 \cdot 8$ & $1 \cdot 7$ \\
\hline 1086 & Concomitant squint & $1 \cdot 8$ & $1 \cdot 7$ \\
\hline 1461 & Idiopathic epilepsy & $1 \cdot 8$ & $1 \cdot 8$ \\
\hline 3089 & Idiopathic epilepsy & $1 \cdot 8$ & $1 \cdot 8$ \\
\hline 2851 & Migraine & $1 \cdot 8$ & $1 \cdot 8$ \\
\hline 2802 & Migraine & $1 \cdot 8$ & $1 \cdot 8$ \\
\hline 1406 & Psychoneurosis & $1 \cdot 8$ & $1 \cdot 8$ \\
\hline 2362 & Psychoneurosis & $1 \cdot 8$ & $1 \cdot 8$ \\
\hline 979 & Retrobulbar neuritis & $1 \cdot 8$ & $1 \cdot 8$ \\
\hline *1946 & Idiopathic epilepsy & $1 \cdot 8$ & 1.9 \\
\hline 2365 & Idiopathic epilepsy & $1 \cdot 8$ & 1.9 \\
\hline 2696 & Idiopathic epilepsy & $1 \cdot 8$ & 1.9 \\
\hline 973 & Psychoneurosis & $1 \cdot 8$ & 1.9 \\
\hline 3384 & Psychopathic personality & $1 \cdot 8$ & 1.9 \\
\hline 2717 & Disseminated sclerosis & $1 \cdot 8$ & 1.9 \\
\hline$* 1950$ & Idiopathic epilepsy & 1.9 & $1 \cdot 8$ \\
\hline$* 1176$ & Psychoneurosis & 1.9 & 1.9 \\
\hline *2731 & Psychoneurosis & 1.9 & 1.9 \\
\hline 706 & Vaso-vagal attacks & 1.9 & 1.9 \\
\hline 3615 & Idiopathic epilepsy & 1.9 & 1.9 \\
\hline 1178 & Idiopathic epilepsy & 1.9 & $2 \cdot 0$ \\
\hline 2431 & Idiopathic epilepsy & $1 \cdot 8$ & $2 \cdot 0$ \\
\hline$* 1288$ & Psychoneurosis & $1 \cdot 8$ & $2 \cdot 0$ \\
\hline$* 2916$ & Psychoneurosis & $1 \cdot 8$ & $2 \cdot 0$ \\
\hline 2719 & Idiopathic epilepsy & $2 \cdot 0$ & $1 \cdot 8$ \\
\hline 2287 & Idiopathic epilepsy & $2 \cdot 0$ & 1.9 \\
\hline${ }^{*} 1501$ & Psychoneurosis & $2 \cdot 0$ & 1.9 \\
\hline
\end{tabular}

Ventricular measurements

R.L.V. L.L.V.

\begin{tabular}{cccc}
$\begin{array}{c}\text { Hospital } \\
\text { case no. }\end{array}$ & Clinical diagnosis & $\begin{array}{r}\text { R.L.V. } \\
\text { (cm.) }\end{array}$ & $\begin{array}{c}\text { L.L.V. } \\
(\mathrm{cm} .)\end{array}$ \\
\hline 1506 & Idiopathic epilepsy & $2 \cdot 0$ & $2 \cdot 0$ \\
940 & Idiopathic epilepsy & $2 \cdot 0$ & $2 \cdot 0$ \\
2612 & Idiopathic epilepsy & $2 \cdot 0$ & $2 \cdot 0$ \\
$* 908$ & Psychoneurosis & $2 \cdot 0$ & $2 \cdot 0$ \\
2994 & Psychoneurosis & $2 \cdot 0$ & $2 \cdot 0$ \\
3144 & Psychoneurosis & $2 \cdot 0$ & $2 \cdot 0$ \\
2028 & Idiopathic epilepsy & $2 \cdot 1$ & $2 \cdot 1$ \\
2885 & Idiopathic epilepsy & $2 \cdot 2$ & $2 \cdot 2$ \\
\multicolumn{4}{c}{ * Denotes the incidence of a trivial head injury. }
\end{tabular}

of the two lateral ventricles were similar, except for the normal anatomical variations in the development of the occipital horns.

It will be observed that in no case was there a difference of more than $2 \mathrm{~mm}$. between the measurements of the two ventricular bodies and that in as many as 28 cases the ventricular measurements were equal on the two sides. It was therefore considered justifiable to assume that, in the absence of gross cranial asymmetry, a variation in measurement between the two ventricular bodies of more than $2 \mathrm{~mm}$. was significant. The upper limit of normality can only be fixed arbitrarily, but, as in only 2 out of the 50 cases was the oblique diameter of the body of the ventricle greater than $2 \mathrm{~cm}$., figures greater than this were taken to indicate dilatation.

\section{Measurement of the Third Ventricle}

The maximum breadth of the third ventricle, as seen in the antero-posterior brow-up projection, was measured in every case. Considerable variation was found to occur in both the head injury and in the control series. There did not appear to us to be any trend towards increased measurement in cases with ventricular dilation. It was therefore decided to regard the breadth of the third ventricle as having too wide a normal range of variation to afford any significant information. This accords with a similar conclusion reached by Fischer (1927).

\section{General}

\section{Analysis of Case Material}

On the basis of the above standards of normality we found evidence of enlargement of the lateral 
ventricles in 69 out of the 100 cases, as opposed to 2 out of 50 cases in the control series. In some cases the enlargement involved only a part of the lateral ventricle (focal dilatations), whereas in others the whole of the lateral ventricle was affected (generalized dilatations). As this suggested that the ventricular dilatations might have been produced by more than one mechanism, we arranged our 100 cases into the following four radiological groups according to the type and extent of the ventricular changes:

(1) Those without dilatation of the ventricles. Table III. (Normal encephalograms) 31 cases
(2) Those with focal dilatation of part of the ventricular system. Table IV. (Focal $\begin{array}{llllll}\text { dilatation }) & \ldots & \ldots & \ldots & \ldots & 15 \text { cases }\end{array}$

(3) Those with generalized dilatations of the lateral ventricles :

(a) Of one ventricle only. Table V. (Unilateral generalized dilatation). .

(b) Of both ventricles. Table VI. (Bilateral generalized dilatation). . . . . . . 26 ,

(4) Those with focal dilatations superimposed on generalized dilatations. Table VII. (Focal with generalized dilatation) . . 7 ,

Table III.-Normal Encephalograms

\begin{tabular}{|c|c|c|c|c|c|c|}
\hline No. & Diagnosis & P.T.A. & $\begin{array}{l}\text { R.L.V. } \\
(\mathrm{cm} .)\end{array}$ & $\begin{array}{l}\text { L.L.V. } \\
(\mathrm{cm} .)\end{array}$ & Site & Fractures \\
\hline 1 & $\begin{array}{l}\text { Head injury. Not unconscious. Post- } \\
\text { traumatic syndrome. }\end{array}$ & Nil & $1 \cdot 8$ & $1 \cdot 7$ & Frontal & Nil \\
\hline 2 & $\begin{array}{l}\text { Head injury. Not unconscious. Post- } \\
\text { traumatic epilepsy. }\end{array}$ & Nil & $1 \cdot 9$ & $1 \cdot 9$ & Left temporal & Nil \\
\hline 3 & $\begin{array}{l}\text { Head injury. Not unconscious. Post- } \\
\text { traumatic syndrome. }\end{array}$ & Nil & $1 \cdot 6$ & $1 \cdot 7$ & Frontal & Compound frontal \\
\hline 4 & $\begin{array}{l}\text { Head injury. Not unconscious. Post- } \\
\text { traumatic syndrome. }\end{array}$ & Nil & $1 \cdot 7$ & $1 \cdot 8$ & Occipital & Nil \\
\hline 5 & Post-traumatic epilepsy $\quad \ldots \quad \ldots$ & Minutes & $1 \cdot 8$ & $1 \cdot 9$ & Left side & Nil \\
\hline 6 & $\begin{array}{l}\text { Head injury with residual pyramidal } \\
\text { signs. }\end{array}$ & Minutes & $1 \cdot 8$ & $1 \cdot 9$ & Right parietal & Nil \\
\hline 7 & Post-traumatic epilepsy $\quad \ldots \quad \ldots$ & Minutes & 1.9 & $2 \cdot 0$ & Not known & Nil \\
\hline 8 & $\begin{array}{l}\text { Head injury without residual se- } \\
\text { quelæ. }\end{array}$ & 2 hours & $1 \cdot 7$ & $1 \cdot 9$ & Right frontal & Nil \\
\hline 9 & $\begin{array}{l}\text { Head injury with residual pyramidal } \\
\text { signs. }\end{array}$ & $2 \frac{1}{2}$ hours & $1 \cdot 7$ & $1 \cdot 7$ & Vertex & $\begin{array}{l}\text { Slightly depressed } \\
\text { vertex }\end{array}$ \\
\hline 10 & Post-traumatic syndrome $\quad \ldots \quad \ldots$ & 3 hours & $*$ & $*$ & Right frontal & Nil \\
\hline 11 & Post-traumatic syndrome $\quad \ldots \quad$. & 12 hours & $1 \cdot 8$ & $1 \cdot 8$ & Right frontal & $\begin{array}{l}\text { Compound } \\
\text { depressed }\end{array}$ \\
\hline 12 & Post-traumatic syndrome $\quad . \quad \ldots$ & 12 hours & $1 \cdot 9$ & $1 \cdot 8$ & Not known & Nil \\
\hline 13 & Head injury with cranial nerve palsy & 18 hours & $1 \cdot 9$ & 1.9 & Frontal & $\begin{array}{l}\text { Slightly depressed } \\
\text { frontal }\end{array}$ \\
\hline 14 & Post-traumatic epilepsy $\quad$. & 1 day & $1 \cdot 7$ & $1 \cdot 9$ & Not known & Nil \\
\hline 15 & Post-traumatic syndrome $\quad$. & $1 \frac{1}{2}$ days & $1 \cdot 9$ & $1 \cdot 9$ & Right parietal & $\begin{array}{l}\text { Depressed right } \\
\text { parietal }\end{array}$ \\
\hline 16 & Post-traumatic syndrome $\quad$. & 2 days & $1 \cdot 6$ & $1 \cdot 7$ & Right frontal & Nil \\
\hline 17 & Post-traumatic syndrome $\quad \ldots \quad \ldots$ & 2 days & $1 \cdot 6$ & $1 \cdot 6$ & Öccipital & Nil \\
\hline 18 & $\begin{array}{l}\text { Head injury with cranial nerve palsy. } \\
\text { Post-traumatic syndrome. }\end{array}$ & 2 days & $1 \cdot 9$ & $1 \cdot 9$ & $\begin{array}{l}\text { Left frontal and } \\
\text { Right occipital }\end{array}$ & $\begin{array}{l}\text { Nil } \\
\text { Nil }\end{array}$ \\
\hline 19 & Head injury with cranial nerve palsy & 3 days & $1 \cdot 7$ & $1 \cdot 7$ & Right temporal & Comminuted \\
\hline 20 & Head injury with cranial nerve palsy & 3 days & $1 \cdot 6$ & $1 \cdot 6$ & Mid-frontal & frontal \\
\hline 21 & Post-traumatic epilepsy & 4 days & $1 \cdot 7$ & $1 \cdot 6$ & Left occipital & $\begin{array}{l}\text { Left posterior } \\
\text { parietal }\end{array}$ \\
\hline 22 & $\begin{array}{l}\text { Head injury with hypothalamic and } \\
\text { brain stem signs. Post-traumatic } \\
\text { intellectual deterioration. }\end{array}$ & 5 days & $\begin{array}{l}1 \cdot 6 \\
1 \cdot 7 \\
1 \cdot 6\end{array}$ & $\begin{array}{l}1 \cdot 6 \\
1 \cdot 7 \\
1 \cdot 6\end{array}$ & Not known & Nil \\
\hline 23 & Post-traumatic syndrome $\quad . \quad \ldots$ & 5 days & $1 \cdot 6$ & $1 \cdot 6$ & Left frontal & Left frontal \\
\hline 24 & Head injury with cranial nerve palsy & 5 days & $1 \cdot 6$ & $1 \cdot 6$ & Right frontal & $\begin{array}{l}\text { Comminuted } \\
\text { frontal }\end{array}$ \\
\hline 25 & $\begin{array}{l}\text { Post - traumatic syndrome. Optic } \\
\text { radiation distrubance. }\end{array}$ & $1 \frac{1}{2}$ weeks & $2 \cdot 0$ & $2 \cdot 0$ & Left occipital & $\begin{array}{l}\text { Depressed left } \\
\text { occipital }\end{array}$ \\
\hline 26 & Post-traumatic syndrome $\ldots . .$. & $1 \frac{1}{2}$ weeks & $1 \cdot 8$ & 1.9 & Not known & Nil \\
\hline 27 & $\begin{array}{l}\text { Post-traumatic intellectual deteriora- } \\
\text { tion. }\end{array}$ & $2 \frac{1}{2}$ weeks & $1 \cdot 6$ & $1 \cdot 6$ & Left temporal & Left temporal \\
\hline $\begin{array}{l}28 \\
29\end{array}$ & Head injury with brain stem signs .. & 3 weeks & 1.9 & 1.9 & Not known & $\mathrm{Nil}$ \\
\hline 29 & $\begin{array}{l}\text { Post-traumatic intellectual deteriora- } \\
\text { tion. }\end{array}$ & 3 weeks & $1 \cdot 8$ & $1 \cdot 8$ & ? Occipital & Nil \\
\hline 30 & Head injury with brain stem signs .. & $\begin{array}{l}4 \text { weeks } \\
3 \text { weeks }\end{array}$ & $1 \cdot 8$ & $1 \cdot 8$ & ? Occipital & Nil \\
\hline 31 & $\begin{array}{l}\text { Head injury with cranial nerve palsy. } \\
\text { Post-traumatic syndrome. }\end{array}$ & $1 \frac{1}{2}$ months & $1 \cdot 6$ & $1 \cdot 7$ & Left side & $\begin{array}{l}\text { Mu!tiple left } \\
\text { parietal }\end{array}$ \\
\hline
\end{tabular}

* Air was present in the cavum septi pellucidi, separating and angulating the ventricular bodies, which were, however, of normal size and outline. It was felt that the standard measurements were not applicable in such a case. 

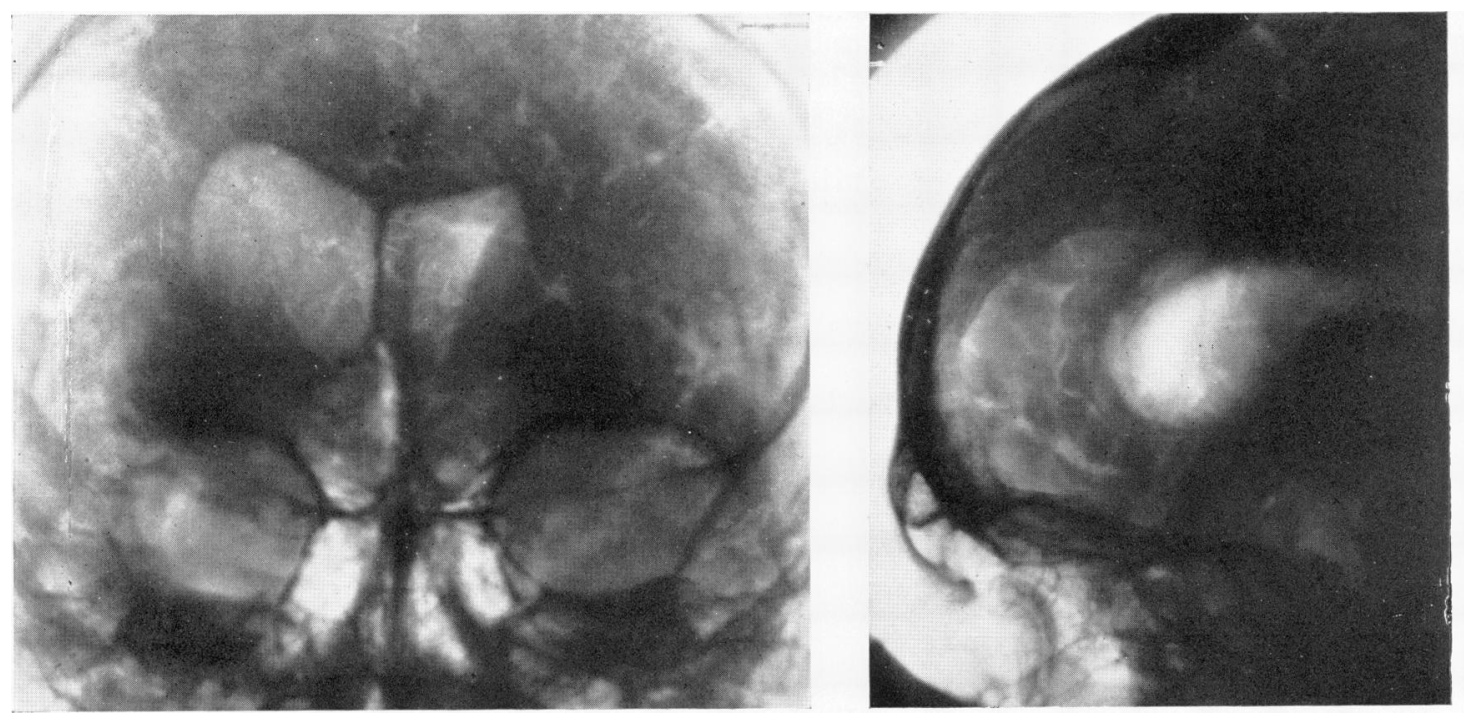

FIG. 3.-Focal dilatation. Case No. 35. Focal dilatation of the anterior horn of the right lateral ventricle.
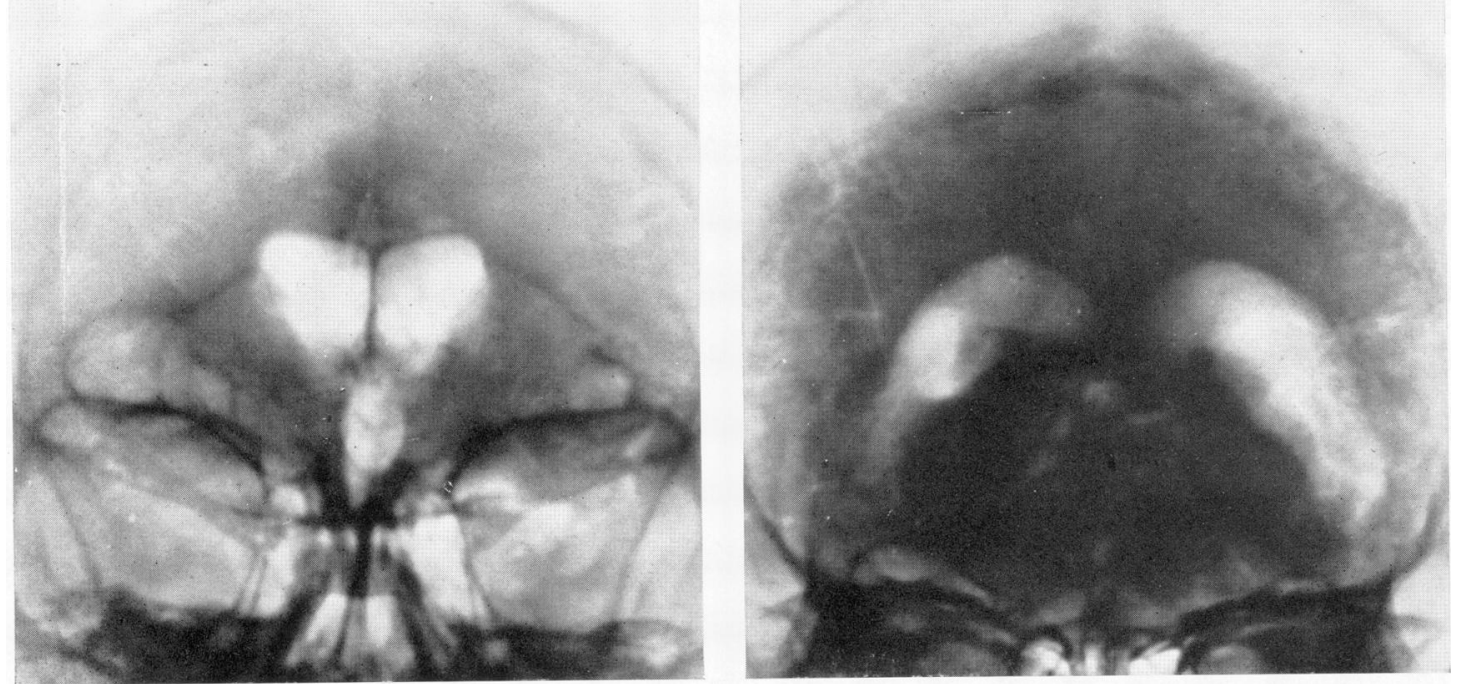

FIG. 4.-Focal dilatation. Case No. 43. Focal dilatation of the trigone of the left lateral ventricle.

These figures establish that ventricular dilatation supervenes in a high proportion of cases after head injury. Our series, however, constitutes a highly selected group of cases in that, with one exception, they all showed either some residual complication due to the head injury or else some unusual feature during the acute stage, such as prolonged posttraumatic confusion or epileptic attacks, which necessitated detailed investigation (see later). This exception, Case 8, was our only example of the common straightforward type of head injury with short duration of unconsciousness and subsequent rapid and complete recovery, and had a normal encephalogram. Consequently our observations are based almost entirely on the more complicated cases which are more likely to show abnormalities. We have evidence, however, to suggest that ventricular dilatation seldom supervenes after trivial injuries, whereas it occurs much more frequently after severe head injuries.

In order to elucidate the factors responsible for the enlargement of the ventricles, we attempted to correlate the type and extent of the ventricular dilatation with the severity of the head injury as 
judged by clinical standards, with the site of injury, and with the time after injury when the dilatation became apparent.

It is often difficult to measure clinically the severity of brain damage resulting from injury, as sometimes even severe destruction of brain tissue may occur without much upset to the patient. Two criteria, which are often used clinically for assessing the severity of head injuries, are the duration of the post-traumatic amnesia and the amount of blood in the cerebro-spinal fluid in the acute phase of the injury. Neither of these criteria is entirely reliable, because sometimes the brain may be seriously damaged without loss of consciousness resulting, or without the appearance of blood in the cerebro-spinal fluid. Of these two criteria the duration of the post-traumatic amnesia is the more easily obtained and was estimated in each of our cases. In Table VIII the frequency and the degree of the ventricular dilatation in our cases is analysed with regard to the length of the post-traumatic amnesia. The table shows that the frequency of ventricular dilatation rises steadily with increasing duration of the post-traumatic amnesia, from 33 per cent. in cases where there was no post-traumatic amnesia to 92 per cent. in cases where the posttraumatic amnesia extended longer than a month. Moreover the extent to which the ventricles dilated also seemed to increase with the duration of the posttraumatic amnesia. Thus out of 18 cases in which the post-traumatic amnesia was under 1 day, 2 (11 per cent.) had focal dilatation and 3 cases ( 17 per cent.) had generalized bilateral dilatation: out of 13 cases in which the post-traumatic amnesia ex-

Table IV.-Focal Dilatations

\begin{tabular}{|c|c|c|c|c|c|c|c|}
\hline No. & Diagnosis & P.T.A. & $\begin{array}{l}\text { R.L.V. } \\
\text { (cm.) }\end{array}$ & $\begin{array}{l}\text { L.L.V. } \\
(\mathrm{cm} .)\end{array}$ & Local changes & Site & Fractures \\
\hline 32 & $\begin{array}{l}\text { Head injury with cranial nerve } \\
\text { palsies. }\end{array}$ & 12 hours & $1 \cdot 8$ & $1 \cdot 9$ & $\begin{array}{l}\text { Dilatation left } \\
\text { anterior horn }\end{array}$ & Frontal & Left frontal \\
\hline 33 & $\begin{array}{l}\text { Head injury without residual } \\
\text { sequelæ. }\end{array}$ & 1 day & $1 \cdot 6$ & $1 \cdot 6$ & $\begin{array}{l}\text { Dilatation left } \\
\text { anterior horn }\end{array}$ & Left frontal & Left frontal \\
\hline 34 & $\begin{array}{l}\text { Head injury with residual } \\
\text { pyramidal signs. Post-trau- } \\
\text { matic intellectual deteriora- } \\
\text { tion. }\end{array}$ & 4 days & $1 \cdot 8$ & $1 \cdot 8$ & $\begin{array}{l}\text { Dilatation right } \\
\text { temporal horn } \\
\text { and vestibule }\end{array}$ & Right side & $\begin{array}{l}\text { Depressed right } \\
\text { temporal }\end{array}$ \\
\hline 35 & Post-traumatic epilepsy $\quad$.. & 4 days & $2 \cdot 4$ & $2 \cdot 4 *$ & $\begin{array}{l}\text { Gross dilatation } \\
\text { right anterior } \\
\text { horn and both } \\
\text { anterior bodies }\end{array}$ & Right frontal & $\begin{array}{l}\text { Right frontal } \\
\text { compound } \\
\text { comminuted }\end{array}$ \\
\hline 36 & $\begin{array}{l}\text { Post - traumatic syndrome. } \\
\text { Post-traumatic epilepsy. }\end{array}$ & I week & $1 \cdot 8$ & $1 \cdot 8$ & $\begin{array}{l}\text { Both anterior } \\
\text { horns dilated; } \\
\text { right more } \\
\text { than left }\end{array}$ & Frontal & $\begin{array}{l}\text { Depressed } \\
\text { frontal }\end{array}$ \\
\hline 37 & Post-traumatic syndrome $\quad$. & 1 week & $1 \cdot 8$ & $1 \cdot 8$ & $\begin{array}{l}\text { Dilated right } \\
\text { vestibule }\end{array}$ & Not known & $\begin{array}{l}\text { Diastasis right } \\
\text { lambdoid }\end{array}$ \\
\hline 38 & $\begin{array}{l}\text { Post - traumatic syndrome. } \\
\text { Head injury with cranial } \\
\text { nerve palsies }\end{array}$ & $1 \frac{1}{2}$ weeks & $2 \cdot 0$ & $1 \cdot 7$ & $\begin{array}{l}\text { Dilated right } \\
\text { anterior horn }\end{array}$ & Right frontal & Right frontal \\
\hline 39 & $\begin{array}{l}\text { Post-traumatic intellectual de- } \\
\text { terioration. }\end{array}$ & $1 \frac{1}{2}$ weeks & $1 \cdot 8$ & $1 \cdot 8$ & $\begin{array}{l}\text { Dilated right } \\
\text { anterior horn }\end{array}$ & Right frontal & Right frontal \\
\hline 40 & $\begin{array}{l}\text { Head injury with residual } \\
\text { pyramidal signs. Post-trau- } \\
\text { matic epilepsy. }\end{array}$ & $2 \frac{1}{2}$ weeks & $\begin{array}{l}1 \cdot 8 \\
1 \cdot 8\end{array}$ & $\begin{array}{l}2 \cdot 0 \\
2 \cdot 0\end{array}$ & $\begin{array}{l}\text { Dilated left an- } \\
\text { terior horn } \\
\text { and right } \\
\text { vestibule }\end{array}$ & Multiple & Right occipital \\
\hline 41 & $\begin{array}{l}\text { Head injury with residual } \\
\text { brain stem signs. }\end{array}$ & 2-3 weeks & $\begin{array}{l}1 \cdot 9 \\
2 \cdot 0\end{array}$ & $\begin{array}{l}2 \cdot 4 \\
2 \cdot 4\end{array}$ & $\begin{array}{l}\text { Dilatation left } \\
\text { anterior body } \\
\text { and anterior } \\
\text { horn }\end{array}$ & Left vertex & Nil \\
\hline 42 & $\begin{array}{l}\text { Head injury with residual } \\
\text { pyramidal signs. }\end{array}$ & 3-4 weeks & $1 \cdot 9$ & $1 \cdot 8$ & $\begin{array}{l}\text { Slight dilata- } \\
\text { tion left vesti- } \\
\text { bule }\end{array}$ & Right temporal & Nil \\
\hline 43 & $\begin{array}{l}\text { Head injury with residual } \\
\text { pyramidal signs. Post-trau- } \\
\text { matic intellectual deteriora- } \\
\text { tion. }\end{array}$ & 1 month & $1 \cdot 8$ & $1 \cdot 8$ & $\begin{array}{l}\text { Slight dilata- } \\
\text { tion left vesti- } \\
\text { bule and tem- } \\
\text { poral horn }\end{array}$ & Right parietal & Right sided \\
\hline 44 & $\begin{array}{l}\text { Head injury with residual } \\
\text { pyramidal signs. }\end{array}$ & 1 month & $1 \cdot 6$ & $2 \cdot 0$ & $\begin{array}{l}\text { Dilatation left } \\
\text { anterior body } \\
\text { and anterior } \\
\text { horn }\end{array}$ & Left frontal & Nil \\
\hline 45 & $\begin{array}{l}\text { Head injury with residual } \\
\text { pyramidal signs. Dysphasia. } \\
\text { Post - traumatic intellectual } \\
\text { deterioration. }\end{array}$ & $1 \frac{1}{2}$ months & $1 \cdot 8$ & $2 \cdot 1$ & $\begin{array}{l}\text { Dilatation left } \\
\text { anterior body } \\
\text { and anterior } \\
\text { horn }\end{array}$ & Left side & Left parietal \\
\hline 46 & $\begin{array}{l}\text { Post-traumatic intellectual de- } \\
\text { terioration. }\end{array}$ & 2 months & $1 \cdot 7$ & $1 \cdot 7$ & $\begin{array}{r}\text { Dilatation both } \\
\text { anterior horns }\end{array}$ & Left parietal & Nil \\
\hline
\end{tabular}

* The dilatation in this case was essentially focal despite the bilateral enlargement. 
Table V.-Generalized Dilatations-Unilateral

\begin{tabular}{|c|c|c|c|c|c|c|}
\hline No. & Diagnosis & P.T.A. & $\begin{array}{l}\text { R.L.V. } \\
\text { (cm.) }\end{array}$ & $\begin{array}{l}\text { L.L.V. } \\
\text { (cm.) }\end{array}$ & Site & Fractures \\
\hline $\begin{array}{l}47 \\
48\end{array}$ & $\begin{array}{lll}\text { Post-traumatic epilepsy } & \ldots & \\
\text { Post-traumatic syndrome }\end{array}$ & $\begin{array}{c}\text { Nil } \\
\text { Minutes }\end{array}$ & $\begin{array}{l}1.9 \\
1.9\end{array}$ & $\begin{array}{l}2 \cdot 5 \\
2 \cdot 3\end{array}$ & $\begin{array}{c}\text { Occipital } \\
\text { Right parietal }\end{array}$ & $\begin{array}{l}\text { Nil } \\
\text { Nil }\end{array}$ \\
\hline 49 & $\begin{array}{l}\text { Head injury with optic-radiation dis- } \\
\text { turbances. }\end{array}$ & Minutes & 1.9 & $2 \cdot 2$ & Right occipital & Compound right \\
\hline 50 & $\begin{array}{l}\text { Post-traumatic intellectual deteriora- } \\
\text { tion. }\end{array}$ & $\begin{array}{l}4 \text { days } \\
2 \frac{1}{2} \text { days }\end{array}$ & $1 \cdot 6$ & $2 \cdot 3$ & Left parietal & Nil \\
\hline 51 & $\begin{array}{l}\text { Head injury with residual pyramidal } \\
\text { signs. Post-traumatic syndrome. }\end{array}$ & 3 days & $\begin{array}{l}2 \cdot 3 \\
2 \cdot 3\end{array}$ & $\begin{array}{l}1 \cdot 8 \\
2 \cdot 0\end{array}$ & Not known & Nil \\
\hline 52 & Head injury with cranial nerve palsy & 3 days & 1.8 & $2 \cdot 4$ & Occipital & Nil \\
\hline 53 & $\begin{array}{l}\text { Post-traumatic intellectual deteriora- } \\
\text { tion. }\end{array}$ & 1 week & $2 \cdot 1$ & $1 \cdot 7$ & Right frontal & Nil \\
\hline 54 & Post-traumatic syndrome $\quad \ldots \quad \ldots$ & 1 week & $1 \cdot 8$ & $2 \cdot 5$ & Left frontal & Nil \\
\hline 55 & $\begin{array}{l}\text { Head injury with residual pyramidal } \\
\text { signs. }\end{array}$ & $1 \frac{1}{2}$ weeks & $2 \cdot 2$ & $1 \cdot 8$ & Right parietal & Right parietal \\
\hline 56 & $\begin{array}{l}\text { Head injury with residual pyramidal } \\
\text { signs and cranial nerve palsies. }\end{array}$ & 2 weeks & $2 \cdot 0$ & $1 \cdot 8$ & Right parietal & Right parietal \\
\hline 57 & $\begin{array}{l}\text { Head injury with residual pyramidal } \\
\text { signs. Post-traumatic epilepsy. }\end{array}$ & 2 weeks & $2 \cdot 0$ & $3 \cdot 0$ & ? Left side & ? Left parietal \\
\hline 58 & $\begin{array}{l}\text { Post-traumatic intellectual deteriora- } \\
\text { tion. }\end{array}$ & 2 weeks & $2 \cdot 0$ & $2 \cdot 3$ & Not known & Nil \\
\hline 59 & $\begin{array}{l}\text { Head injury with residual pyramidal } \\
\text { signs. }\end{array}$ & 2 weeks & $2 \cdot 0$ & $2 \cdot 3$ & Frontal & Basal \\
\hline 60 & $\begin{array}{l}\text { Head injury with residual pyramidal } \\
\text { signs. Post-traumatic epilepsy. }\end{array}$ & 2 weeks & $\begin{array}{l}2 \cdot 0 \\
2 \cdot 0\end{array}$ & $\begin{array}{l}1 \cdot 7 \\
1.7\end{array}$ & Right side & Right temporal \\
\hline 61 & Head injury without residual sequelæ & 3 weeks & $1 \cdot 9$ & $2 \cdot 5$ & Right temporal & Right frontal \\
\hline 62 & $\begin{array}{l}\text { Head injury with residual pyramidal } \\
\text { signs. }\end{array}$ & 3 weeks & $1 \cdot 7$ & $2 \cdot 1$ & Left temporal & \\
\hline 63 & $\begin{array}{l}\text { Post-traumatic intellectual deteriora- } \\
\text { tion. }\end{array}$ & 3 weeks & 1.5 & $2 \cdot 0$ & Left parietal & Left parietal \\
\hline 64 & $\begin{array}{l}\text { Post-traumatic syndrome. Post- } \\
\text { traumatic epilepsy. }\end{array}$ & 3 weeks & $1 \cdot 7$ & $2 \cdot 0$ & Right parietal & Nil \\
\hline $\begin{array}{l}65 \\
66\end{array}$ & $\begin{array}{l}\text { Head injury without residual sequelæ } \\
\text { Head injury without residual sequelæ }\end{array}$ & $\begin{array}{l}3-4 \text { weeks } \\
1 \frac{1}{2} \text { months }\end{array}$ & $\begin{array}{l}2 \cdot 0 \\
1.6\end{array}$ & $\begin{array}{l}2 \cdot 3 \\
2 \cdot 0\end{array}$ & $\begin{array}{l}\text { Frontal } \\
\text { Not known }\end{array}$ & $\begin{array}{l}\text { Nil } \\
\text { Nil }\end{array}$ \\
\hline $\begin{array}{l}00 \\
67 \\
\end{array}$ & Post-traumatic syndrome .. .. & 3 weeks & $\begin{array}{l}1.0 \\
1.9\end{array}$ & $2 \cdot 1$ & Not known & Nil \\
\hline
\end{tabular}
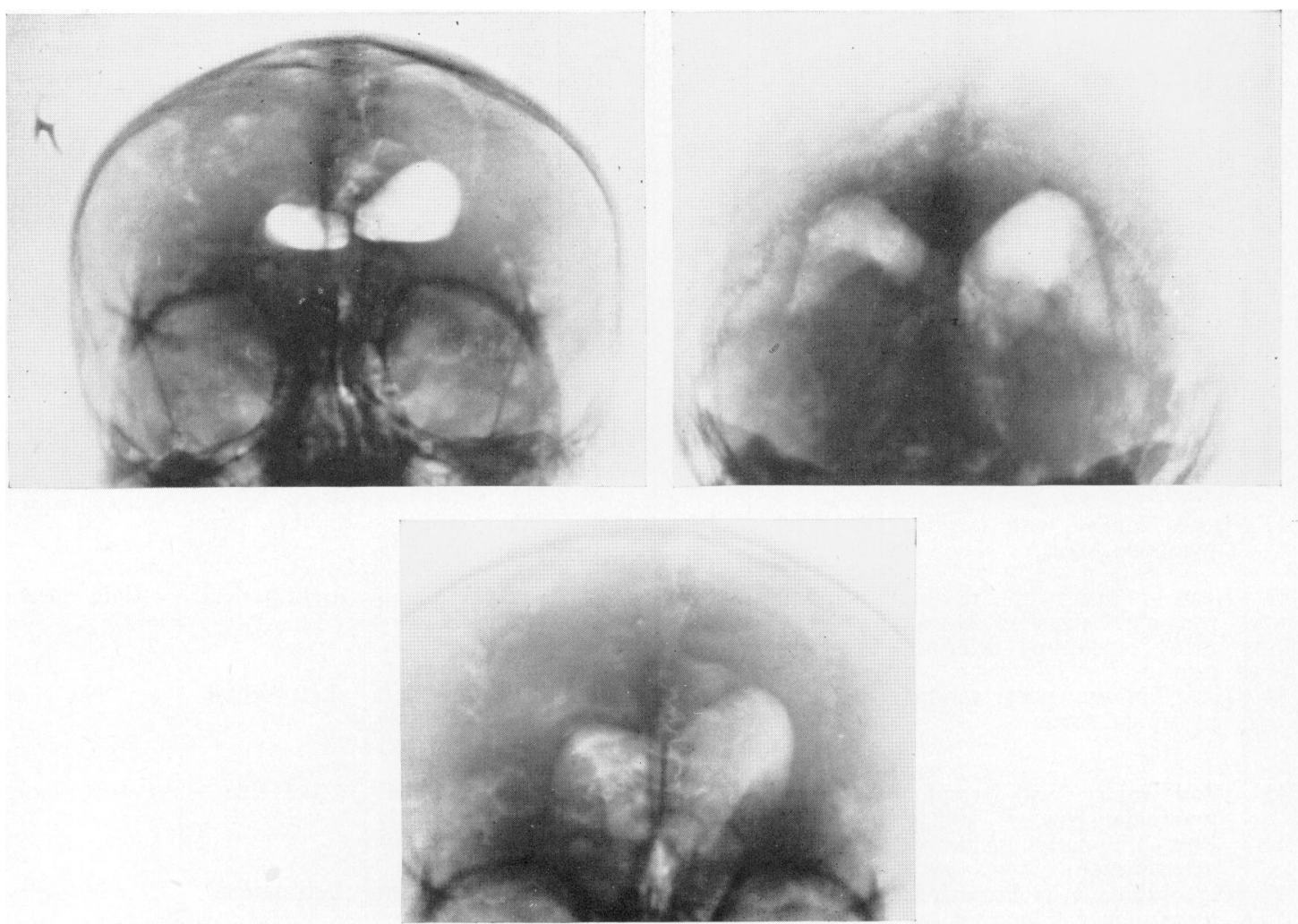

FIG. 5.-Unilateral general dilatation. Case No. 57. General dilatation of the left lateral ventricle without focal changes or enlargement of the right lateral ventricle. 

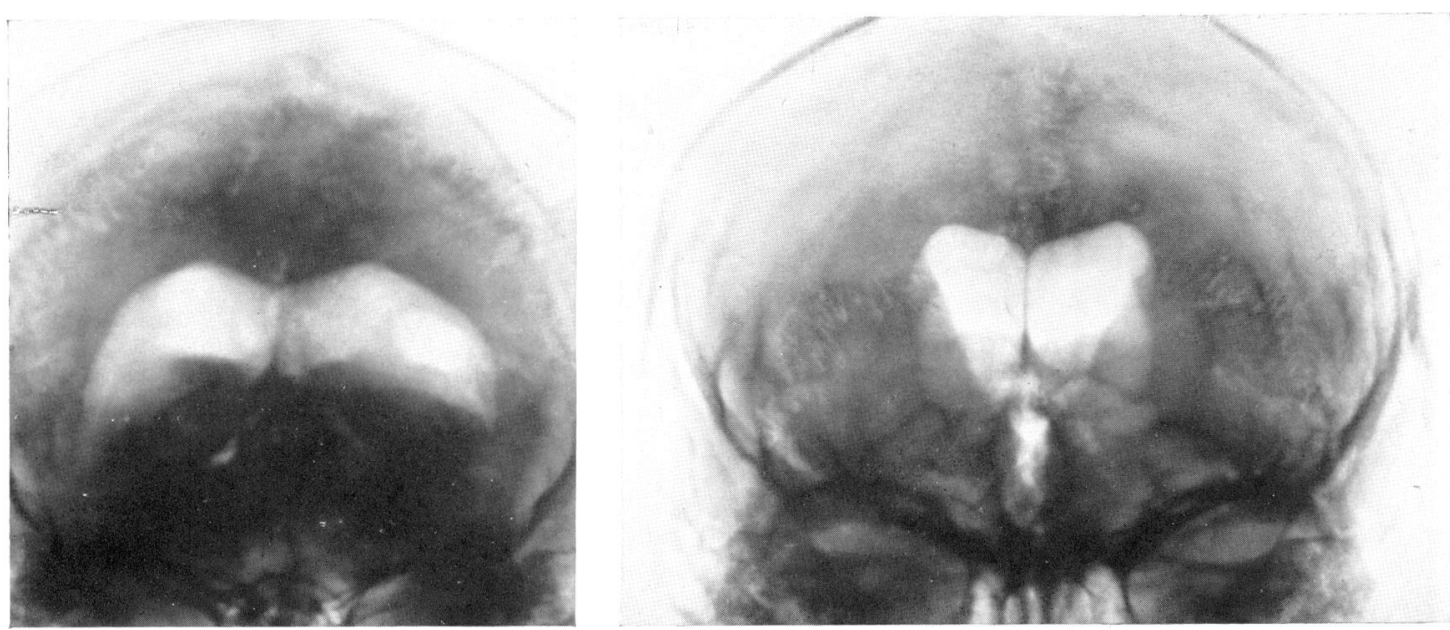

FIG. 6.-Bilaieral general dilatation. Case No. 81. Dilatation of both lateral ventricles, the left being somewhat more affected.

Table Vi.-Generalized Dilatations-Bilateral

\begin{tabular}{|c|c|c|c|c|c|c|}
\hline No. & Diagnosis & P.T.A. & $\begin{array}{l}\text { R.L.V. } \\
(\mathrm{cm} .)\end{array}$ & $\begin{array}{l}\text { L.L.V. } \\
\text { (cm.) }\end{array}$ & Site & Fractures \\
\hline 68 & $\begin{array}{l}\text { Head injury without unconsciousness. } \\
\text { Head injury with pyramidal resi- } \\
\text { dual signs. }\end{array}$ & Nil & $2 \cdot 5$ & $2 \cdot 1$ & Mid-vertex & Depressed vertical \\
\hline 69 & $\begin{array}{l}\text { Head injury with residual pyramidal } \\
\text { signs. }\end{array}$ & 3 minutes & $2 \cdot 5$ & $2 \cdot 0$ & Left side & $\begin{array}{l}\text { Diastasis left } \\
\text { coronal }\end{array}$ \\
\hline 70 & Post-traumatic syndrome $\ldots . \quad \ldots$ & 1 day & $2 \cdot 4$ & $2 \cdot 1$ & Right side & Nil \\
\hline 71 & $\begin{array}{l}\text { Post-traumatic intellectual deteriora- } \\
\text { tion. }\end{array}$ & 1 day & $2 \cdot 3$ & $3 \cdot 2$ & Right frontal & Nil \\
\hline 72 & $\begin{array}{l}\text { Post-traumatic intellectual deteriora- } \\
\text { tion. }\end{array}$ & 4 days & $2 \cdot 7$ & $2 \cdot 8$ & Left frontal & Nil \\
\hline 73 & $\begin{array}{l}\text { Head injury with cranial nerve palsy. } \\
\text { Post-traumatic epilepsy. }\end{array}$ & 4-5 days & $2 \cdot 4$ & $2 \cdot 5$ & Right side & Right parietal \\
\hline 74 & Post-traumatic epilepsy $\quad \ldots \quad \ldots$ & 1 week & $2 \cdot 4$ & $2 \cdot 4$ & Right temporal & Right temporal \\
\hline 75 & $\begin{array}{l}\text { Head injury with optic radiation dis- } \\
\text { turbance. Post-traumatic epilepsy. }\end{array}$ & 1 week & $2 \cdot 3$ & $2 \cdot 3$ & Not known & Left parietal \\
\hline 76 & Head injury without residual sequelæ & $1 \frac{1}{2}$ weeks & $\begin{array}{l}2 \cdot 1 \\
2 \cdot 3\end{array}$ & $\begin{array}{l}2 \cdot 4 \\
2 \cdot 5\end{array}$ & Right parietal & Nil \\
\hline 77 & $\begin{array}{l}\text { Post-traumatic intellectual deteriora- } \\
\text { tion. Post-traumatic epilepsy. }\end{array}$ & 2 weeks & $2 \cdot 4$ & $2 \cdot 4$ & Not known & Nil \\
\hline 78 & $\begin{array}{l}\text { Head injury with cranial nerve pal- } \\
\text { sies. Post-traumatic syndrome. }\end{array}$ & 2 weeks & $2 \cdot 3$ & $2 \cdot 3$ & Not known & Right parietal \\
\hline 79 & $\begin{array}{l}\text { Head injury with residual pyramidal } \\
\text { signs. Post-traumatic intellectual } \\
\text { deterioration. }\end{array}$ & 2-3 weeks & $2 \cdot 1$ & $2 \cdot 3$ & Not known & Nil \\
\hline 80 & $\begin{array}{l}\text { Post-traumatic intellectual deteriora- } \\
\text { tion. }\end{array}$ & 3 weeks & 2.5 & $3 \cdot 0$ & Frontal & Left parietal \\
\hline 81 & Post-traumatic syndrome $\quad \ldots \quad \ldots$ & 3 weeks & $2 \cdot 6$ & $3 \cdot 1$ & Not known & Occipital \\
\hline 82 & $\begin{array}{l}\text { Head injury with residual pyramidal } \\
\text { signs. Dysphasia and cranial nerve } \\
\text { palsies. Post-traumatic epilepsy. }\end{array}$ & 3 weeks & $2 \cdot 8$ & $2 \cdot 8$ & Not known & Right parietal \\
\hline 83 & $\begin{array}{l}\text { Head injury with cranial nerve pal- } \\
\text { sies. Post-traumatic syndrome. }\end{array}$ & 4 weeks & $2 \cdot 1$ & $2 \cdot 6$ & Occipital? & $\begin{array}{l}\text { Left sided } \\
\text { multiple }\end{array}$ \\
\hline 84 & $\begin{array}{l}\text { Head injury with residual pyramidal } \\
\text { signs. }\end{array}$ & +1 month & $2 \cdot 0$ & $2 \cdot 4$ & Right side? & Nil \\
\hline 85 & $\begin{array}{l}\text { Post-traumatic intellectual deteriora- } \\
\text { tion. }\end{array}$ & +1 month & $2 \cdot 2$ & $2 \cdot 7$ & Not known & Not known \\
\hline 86 & $\begin{array}{l}\text { Post-traumatic intellectual deteriora- } \\
\text { tion. }\end{array}$ & +1 month & $2 \cdot 2$ & $2 \cdot 7$ & Frontal & Nil \\
\hline $\begin{array}{l}87 \\
88\end{array}$ & Head injury without residual sequelie & +1 month & $2 \cdot 1$ & $2 \cdot 3$ & Right frontal & Right frontal \\
\hline $\begin{array}{l}88 \\
89\end{array}$ & $\begin{array}{l}\text { Head injury without residual sequela } \\
\text { Head injury without residual sequelæe }\end{array}$ & $\begin{array}{l}1 \frac{1}{2} \text { months } \\
1 \frac{1}{2} \text { months }\end{array}$ & $\begin{array}{l}2 \cdot 8 \\
2 \cdot 8\end{array}$ & $\begin{array}{l}2 \cdot 3 \\
3 \cdot 0\end{array}$ & $\begin{array}{l}\text { Frontal } \\
\text { Vertex }\end{array}$ & $\begin{array}{l}\text { Nil } \\
\text { Nil }\end{array}$ \\
\hline 90 & $\begin{array}{l}\text { Post-traumatic intellectual deteriora- } \\
\text { tion. }\end{array}$ & $1 \frac{1}{2}$ months & $2 \cdot 5$ & $2 \cdot 8$ & Right frontal & Nil \\
\hline 91 & $\begin{array}{l}\text { Post-traumatic intellectual deteriora- } \\
\text { tion. }\end{array}$ & $1 \frac{1}{2}$ months & $2 \cdot 1$ & $2 \cdot 2$ & Right side & Maxilla \\
\hline 92 & $\begin{array}{l}\text { Post-traumatic intellectual deteriora- } \\
\text { tion. }\end{array}$ & $\begin{array}{c}+1 \frac{1}{2} \\
\text { months }\end{array}$ & $2 \cdot 5$ & $2 \cdot 7$ & Not known & Nil \\
\hline 93 & $\begin{array}{l}\text { Head injury with residual pyramidal } \\
\text { signs. Post-traumatic intellectual } \\
\text { deterioration. }\end{array}$ & $\begin{array}{l}2-3 \\
\text { months }\end{array}$ & $2 \cdot 8$ & $2 \cdot 5$ & Not known & Right parietal \\
\hline
\end{tabular}



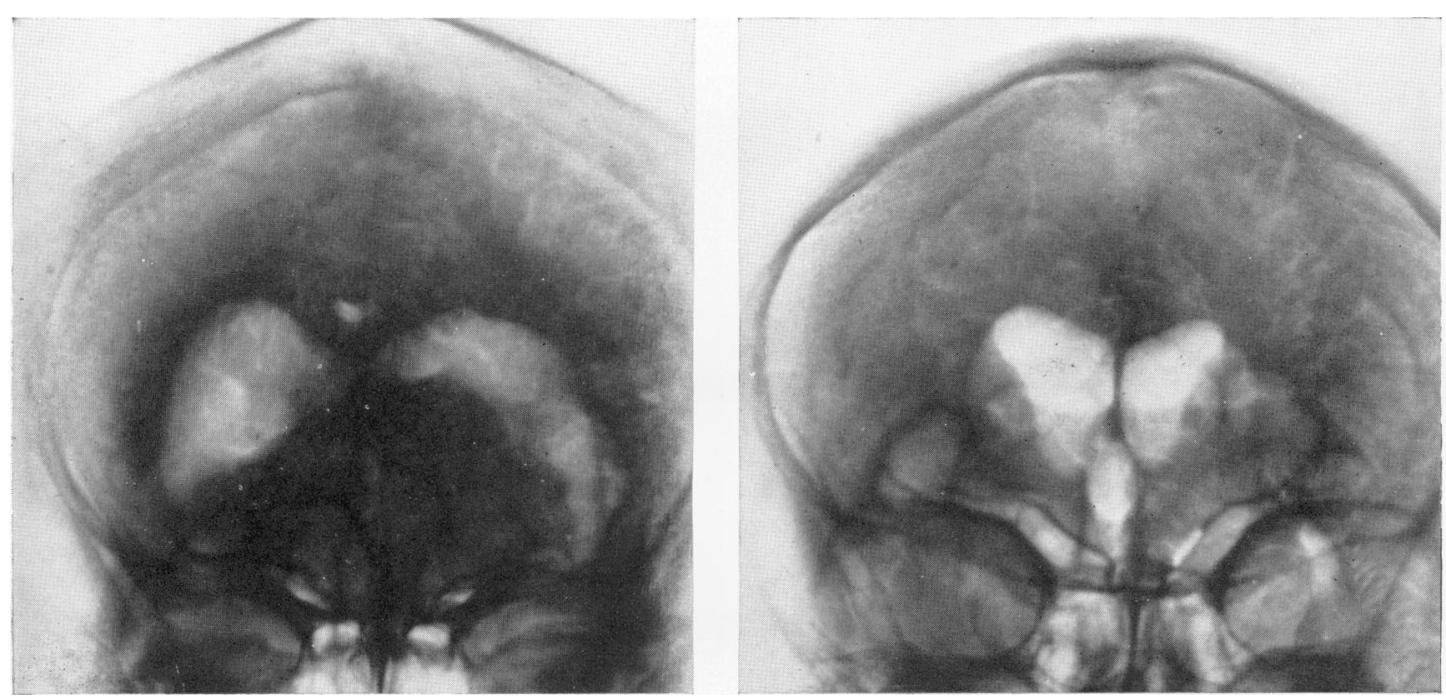

FIG. 7.-Bilateral general dilatation. Case No. 88. Bilateral general dilatation of the lateral ventricles, the right side being more affected than the left but both being enlarged.

Table Vil.-Focal Dilatations Associated with Generalized

\begin{tabular}{|c|c|c|c|c|c|c|c|}
\hline No. & Diagnosis & P.T.A. & $\begin{array}{l}\text { R.L.V. } \\
\text { (cm.) }\end{array}$ & $\begin{array}{l}\text { L.L.V. } \\
\text { (cm.) }\end{array}$ & Local changes & Site & Fractures \\
\hline 94 & Post-traumatic syndrome & 1 day & $2 \cdot 3$ & $2 \cdot 6$ & $\begin{array}{l}\text { Left more than } \\
\text { right through- } \\
\text { out but es- } \\
\text { pecially left } \\
\text { vestibule and } \\
\text { pos te rior } \\
\text { horns }\end{array}$ & Frontal & Basal \\
\hline 95 & $\begin{array}{l}\text { Head injury with residual } \\
\text { pyramidal signs. }\end{array}$ & 4 days & $2 \cdot 0$ & $2 \cdot 7$ & $\begin{array}{l}\text { Relative dilata- } \\
\text { tion of left } \\
\text { frontal and } \\
\text { t e m p o r a l } \\
\text { horns }\end{array}$ & Left frontal & Left frontal \\
\hline 96 & $\begin{array}{l}\text { Head injury with bilateral } 6 \text { th } \\
\text { nerve palsy. }\end{array}$ & 4 days & $2 \cdot 1$ & $2 \cdot 3$ & $\begin{array}{l}\text { Left vestibule } \\
\text { dilated }\end{array}$ & Frontal & Frontal \\
\hline 97 & Post-traumatic epilepsy $\quad \ldots$ & 5 days & $\begin{array}{l}1 \cdot 7 \\
1 \cdot 7\end{array}$ & $\begin{array}{l}2 \cdot 2 \\
2 \cdot 2\end{array}$ & $\begin{array}{l}\text { Left a little }+ \\
\text { anteriorly }\end{array}$ & Frontal & Nil \\
\hline 98 & $\begin{array}{l}\text { Head injury with residual } \\
\text { pyramidal signs. }\end{array}$ & 4 weeks & $2 \cdot 0$ & 1.9 & $\begin{array}{l}\text { Right vestibule } \\
\text { dilated }\end{array}$ & Not known & Nil \\
\hline 99 & $\begin{array}{l}\text { Head injury with residual } \\
\text { pyramidal signs. }\end{array}$ & 4 weeks & $1 \cdot 8$ & $2 \cdot 1$ & $\begin{array}{l}\text { Left vestibule } \\
\text { dilated }\end{array}$ & Occipital & Nil \\
\hline 100 & $\begin{array}{l}\text { Post-traumatic intellectual de- } \\
\text { terioration. }\end{array}$ & $\begin{array}{l}\text { Not as- } \\
\text { sessable }\end{array}$ & $2 \cdot 0$ & $2 \cdot 0$ & $\begin{array}{l}\text { Both anterior } \\
\text { horns }+\end{array}$ & Left frontal & $\begin{array}{l}\text { Depressed } \\
\text { frontal }\end{array}$ \\
\hline
\end{tabular}

Table Vili.-Duration of the Post-Traumatic amnesia in the various Types of Post-Traumatic Ventricular Dilatation

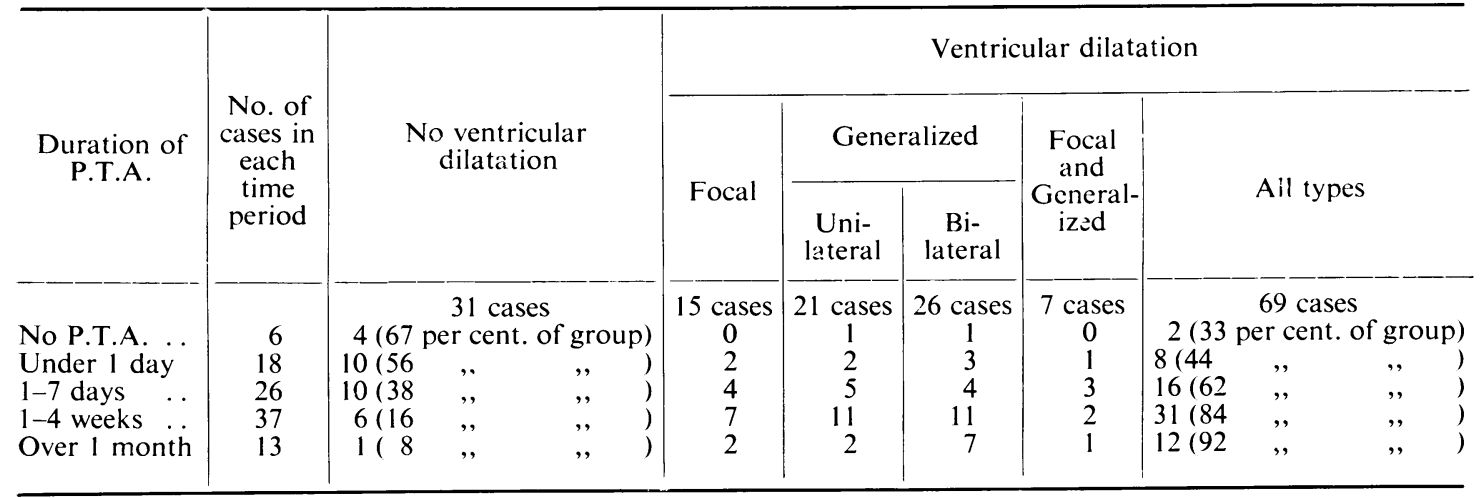



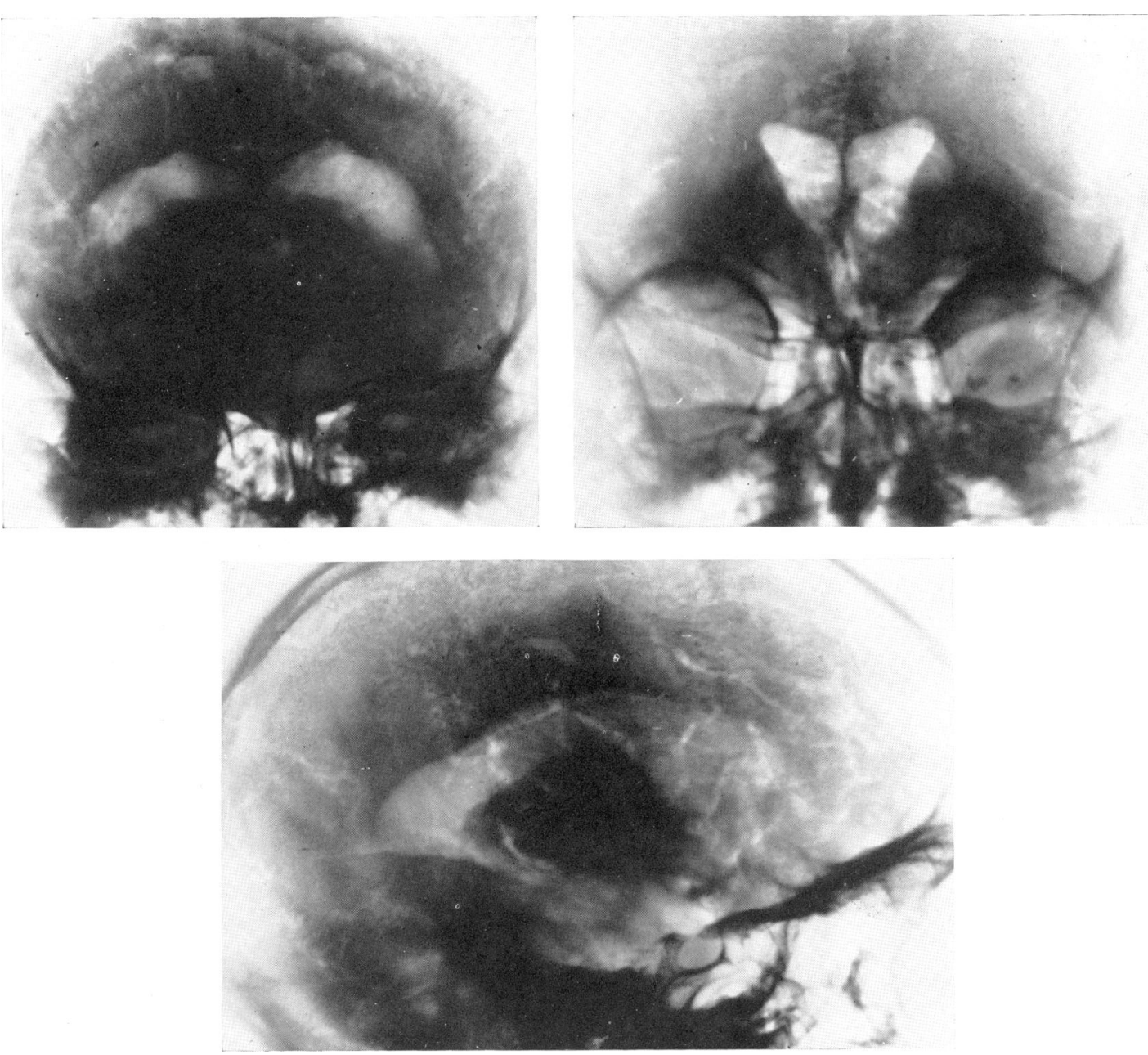

Fig. 8.-Focal dilatation associated with generalized. Case No. 99. An example of general dilatation of the left lateral ventricle with a superimposed focal dilatation of the trigone. The right lateral ventricle is unaifected.

tended longer than a month 2 cases (15 per cent.) had focal dilatation and 7 (54 per cent.) had generalized bilateral dilatation.

Records of the findings on lumbar puncture performed during the first week after injury were available in only 17 cases. In 2 of these the cerebrospinal fluid was normal, whereas in the remaining 15 cases it contained blood. Of the 17 cases, 4, including the two with normal cerebro-spinal fluids, were later shown to have normal ventricular outlines, whereas 13 of those which had had subarachnoid hæmorrhage were later demonstrated to have dilated ventricles. Assuming that blood in the cerebrospinal fluid is an indication of severe head injury, these observations confirm the impression derived from the analysis of the post-traumatic amnesia standards that the more severe degrees of cerebral trauma are apt to be followed by ventricular dilatation.

An analysis of the ventricular changes in relation to the sites at which the violence had been applied did not indicate any causal connexion between the two (Table IX). If, for the moment, fractures of the skull are not taken into account, it would appear from Table IX that both focal dilatations and unilateral or bilateral dilatations of the ventricles are caused just as frequently by injuries delivered in the antero-posterior axis of the skull as they are by injuries delivered from the side. One reason which may account for this apparent lack of correlation between the site of injury and the site and extent of the subsequent ventricular dilatation is that in road accidents, which formed a large proportion of our cases, the skull is often subjected to multiple blows, as Cairns (1941) has shown from a study of the crash helmets of injured motor-cyclists. In 9 cases the dilatation appeared to have been caused by contre-coup; three of these had received occipital injuries, which had resulted in a generalized dilatation of the contralateral ventricle in five cases and focal dilatation in one.

Although the actual site of violence appears to have little connexion with the development of any particular type of ventricular dilatation, the production of a localized fracture of the skull seems to influence the development of a focal dilatation of the ventricle 
Table IX.-Relation of Ventricular Changes to Sites of InJury

\begin{tabular}{|c|c|c|c|c|c|}
\hline \multirow{2}{*}{$\begin{array}{l}\text { Encephalographic } \\
\text { appearances }\end{array}$} & \multirow{2}{*}{$\begin{array}{l}\text { No. of } \\
\text { cases in } \\
\text { each } \\
\text { group }\end{array}$} & \multicolumn{4}{|c|}{ Site of injury to skull } \\
\hline & & $\begin{array}{l}\text { Frontal and } \\
\text { occipital }\end{array}$ & I.ateral & Vertical & Not known \\
\hline Normal ventricles & 31 & $\begin{array}{l}\text { Cases } \\
17 \text { (55 per cent. }\end{array}$ & $\begin{array}{c}\text { Cases } \\
7\end{array}$ (23 per cent. & Cases 13 per cent. & $\begin{array}{c}\text { Cases } \\
6\end{array}$ (19 per cent. \\
\hline $\begin{array}{l}\text { Focal dilatation of ven- } \\
\text { tricles.. }\end{array}$ & 15 & $\begin{array}{rr}7 & \text { (47 per cent. } \\
\text { of group) }\end{array}$ & $\begin{array}{l}6 \text { (40 per cent. } \\
\text { of group) }\end{array}$ & $\begin{array}{l}\text { (7 per cent. } \\
\text { of group }\end{array}$ & $\begin{array}{c}\text { (7 per cent. } \\
\text { of group) }\end{array}$ \\
\hline $\begin{array}{l}\text { Generalized dilatation } \\
\text { of ventricles } \\
\text { (a) Unilateral }\end{array}$ & 47 & $\begin{array}{r}\text { (32 per cent. } \\
\text { of group) } \\
7 \text { (33 per cent. } \\
\text { of group) }\end{array}$ & $\begin{array}{r}\text { (36 per cent. } \\
\text { of group ) } \\
10 \text { (48 per cent. } \\
\text { of group) }\end{array}$ & $\begin{array}{c}2 \quad \text { (4 per cent. } \\
\text { of group) } \\
-\end{array}$ & 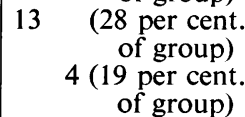 \\
\hline (b) Bilateral $\quad$.. & 26 & 8 (31 per cent. & 7 (27 per cent. & $\begin{array}{l}2 \text { (8 per cent. } \\
\text { of group) }\end{array}$ & $\begin{array}{l}9 \text { (35 per cent. } \\
\text { of group) }\end{array}$ \\
\hline $\begin{array}{l}\text { Focal and generalized } \\
\text { dilatations of ven- } \\
\text { tricles }\end{array}$ & 7 & $\begin{array}{r}\text { (86 per cent. } \\
\text { of group) }\end{array}$ & $\begin{array}{l}114 \text { per cent. } \\
\text { of group) }\end{array}$ & - & - \\
\hline
\end{tabular}

Table X.-Relation of Ventricular Changes to Fracture of Skull.

\begin{tabular}{l} 
Encephalographic appearances \\
\hline
\end{tabular}

(Table X). Thus a fracture of the skull was observed in 15 out of 22 cases ( 68 per cent.) with focal or focal and generalized dilatations of the skulls as opposed to 34 out of 78 cases ( 44 per cent.) in which the ventricles were normal or only showed a uniform enlargement. In the majority of the cases of focal dilatation of the ventricles associated with fracture, the ventricular enlargement occurred immediately beneath the site of fracture ( 9 out of 11 cases). In 2 cases, however, the ventricle on the opposite side had dilated, apparently by contre-coup.

As regards the time of onset of post-traumatic ventricular dilatation, we have evidence which suggests that it is usual for dilatation to commence in less than 3 weeks and that it may reach its maximum within 4 weeks. This evidence is, however, scanty, as encephalography was seldom undertaken during the acute phase of head injury, and, moreover, was usually performed only once in each case. Out of the 69 cases which showed ventricular enlargement, only 7 were examined within a month of injury. Of these, 5, which were examined between 3 and 4 weeks after injury (Cases 32, 43, 53, 59, and 63), showed ventricular dilatation. In one of these (Case 43), which was first examined 1 month after injury, and later re-examined 2 months and $3 \frac{1}{2}$ months after injury, the dilatation did not appear to have progressed after the first examination. In 2 cases (Cases 40 and 76), which were first examined earlier than 3 weeks after injury, some progression in the ventricular changes was subsequently demonstrated at a later examination. In Case 40 the first encephalogram ( 2 weeks after injury), was normal but encephalography repeated at 6 weeks and again at 8 weeks showed that a dilatation of the left anterior horn and right ventricle had appeared between the first and second examinations, but had not increased in size after the 6th week. In the second case (Case 76), slight ventricular enlargement, which was present at $2 \frac{1}{2}$ weeks, appeared to have increased when examined at 5 weeks. We have no cases in our series in which we found evidence of progression of the ventricular enlargement later than 4-6 weeks after injury.

\section{Correlation with symptoms and physical signs}

An analysis of the clinical features of our cases, made in conjunction with the encephalographic findings, shows that the connexion between the degree and type of the ventricular enlargement and the clinical symptoms is not always obvious. In onecase, for example, generalized bilateral ventricular dilatation of considerable degree might (Case 93), be associated with marked residual neurological 
signs, and in another case (Case 88) with none at all. Again, when one ventricle is dilated more than its fellow, the neurological signs present, contrary to the usual observation, may be referable to the side with the smaller or more normal ventricle, whereas the side of the brain with the greater ventricular dilatation gives rise to no symptoms or signs.

Frequently, however, the enlargement of the ventricles could be correlated with neurological features, such as hemiparesis, aphasia, or visual field disturbances, indicating permanent focal damage to the brain. In Table XI the frequency of ventricular dilatation in cases with persistent neurological signs is compared with that in cases without signs of focal cerebral damage. It shows that about 89 per cent. of the cases with persistent focal signs showed ventricular enlargement, as compared with 62 per cent. of the cases in the second group and with 4 per cent. of cases in the control series.

In order to appraise the clinical significance of post-traumatic ventricular dilatation more fully, we arranged our cases into the following clinical groups and attempted to correlate the clinical features with the encephalographic appearances.

(1) Head injury without unconsciousness .. 6 cases

(2) Head injury without residual sequelæ .. 9 ,"

(3) Head injury with residual neurological

$$
\begin{gathered}
\text { signs With pyramidal signs } 24 \text { cases } \\
\text { (a) With hypothalamic and/ } \\
\text { or brain stem signs } 4, \\
\text { (c) With optic radiation dis- } \\
\text { turbances .. } 3, \\
\text { (d) With cranial nerve pal- } \\
\text { sies . . }
\end{gathered}
$$

(4) Post-traumatic syndrome .. ". 29 ,

(5) Post-traumatic intellectual deterioration 24 ,",

(6) Post-traumatic epilepsy .. $\quad$. . $\quad$. 19 ,"

A total of 131 diagnoses thus was made in the 100 cases, multiple diagnoses being made in 30 cases. In each instance the diagnosis was based on the clinical features prevailing after recovery from the head injury and not on the signs present in the acute stage. The allocation of cases to the first three groups and to the last group was straightforward. The assignment of cases to the groups of post-traumatic syndrome and post-traumatic intellectual deterioration was necessarily more arbitrary; those cases in which chronic headaches, often associated with giddiness and fatigue, were prominent were allocated to the first group, and those in which an objective personality change predominated were assigned to the second group. No case was placed in both groups. No separate group was made for cases showing residual sensory signs or, in cases where the dominant hemisphere was damaged, for residual speech disturbances, because in no instance were such signs observed without motor signs, and consequently these cases are already represented in the group showing residual pyramidal signs.

The frequency with which these clinical states were encountered in relation to the various grades of post-traumatic ventricular dilatation is outlined in Table XII. A more detailed study of each group will be given below, but certain important generalizations may here be stressed. Residual pyramidal signs indicative of hemisphere damage are very frequently associated with dilatation of the ventricle on the opposite side of the brain. Primary hypothalamic and brain stem signs, on the other hand, are usually not associated with ventricular dilatation. Cases of post-traumatic syndrome usually have normal or only slightly dilated ventricles, whereas cases of post-traumatic intellectual deterioration often show most marked ventricular dilatation. In all these groups, therefore, an apparent connexion between clinical features and ventricular changes can be made out. On the other hand, no such relationship is evident in cases presenting cranial nerve palsies or post-traumatic epilepsy. Our findings in the groups of head injury without unconsciousness and head injury without residual sequelæ are not applicable to head injuries in general, as our material in these two groups is too highly selected.

A more detailed analysis of each clinical group will now be considered.

\section{Head Injury without Unconsciousness}

There were 6 cases in this group. All had a clear recollection of the accident, and were subsequently investigated between 3 and 15 months later on account of persistent features considered to be due to the injury (post-traumatic syndrome, 3 cases; post-traumatic epilepsy, 2 cases; residual pyramidal signs, 1 case). Four cases showed normal ventricular outlines, and two (Cases 47 and 68) showed some dilatation of the ventricles presumably related to the head injury.

Table XI.-Frequency of Ventricular Dilatation in the Head Injury and Control Series

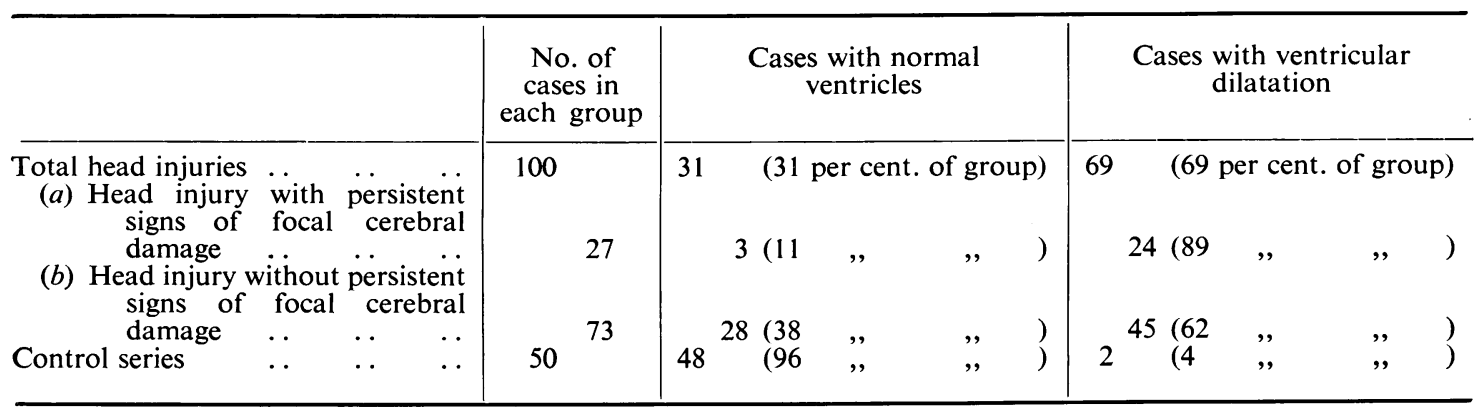


Table XII.-Frequency of the Different Clinical States in the various Types of Post-Traumatic Ventricular Dilatation

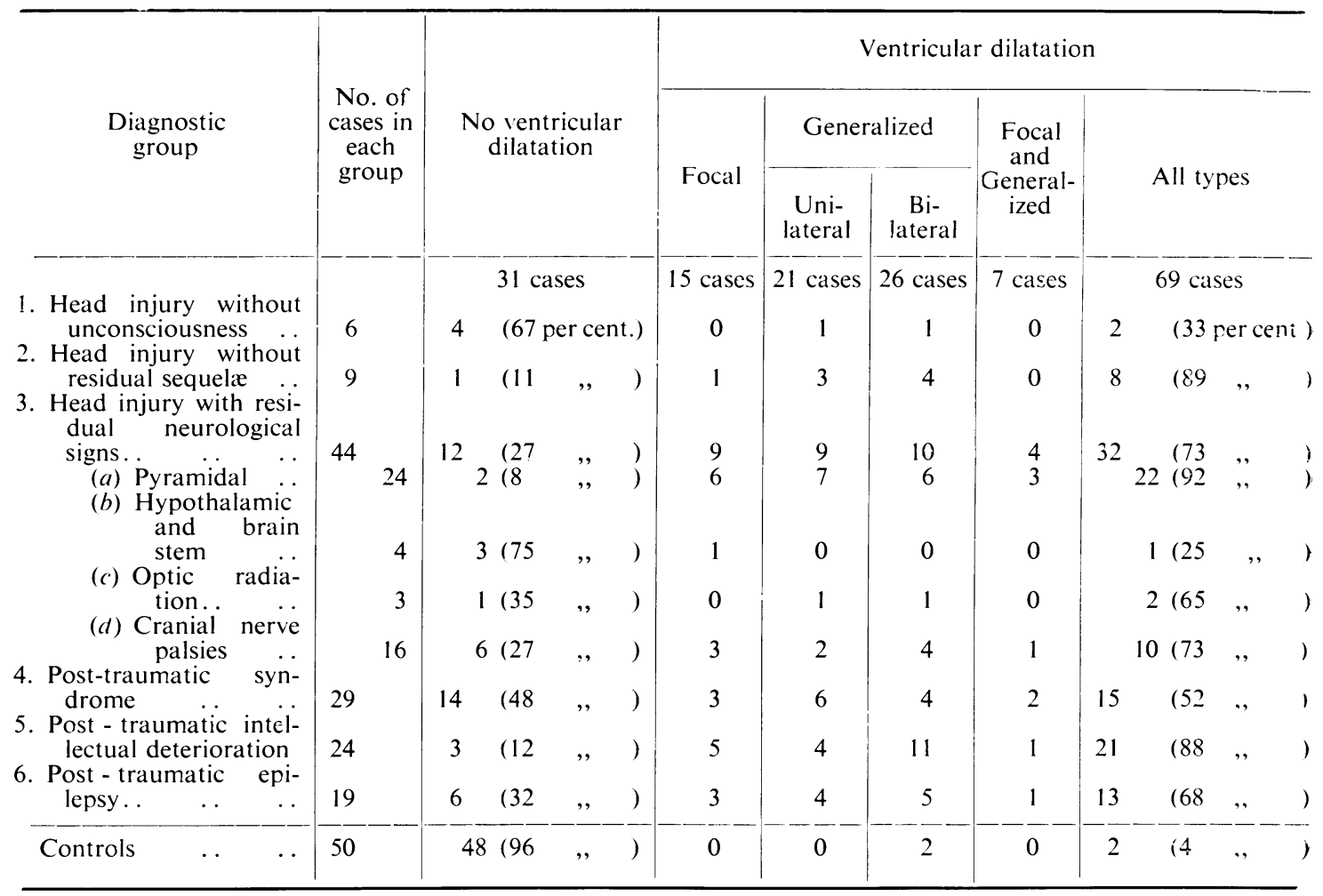

Case 47.- The first of these two cases with ventricular dilatation was a 22-year-old signaller, who fell 12-15 feet backwards from a telegraph pole, striking his head on soft earth without losing consciousness. Four hours later he had a generalized epileptic convulsion and subsequently he had recurrent attacks at intervals of 2 or 3 months. There were no abnormal neurological signs and no previous personal or family history of epilepsy. The electroencephalogram revealed slow high-voltage waves over both hemispheres, suggestive of idiopathic epilepsy, but because symptoms had first occurred so early after the injury the case was considered clinically to be one of post-traumatic epilepsy. Air encephalography demonstrated moderate dilatation of the left lateral ventricle.

Case 68.-The second case was a 39-year-old aircraftsman who received a blow over the vertex when a building collapsed after an explosion. He sustained a small compound depressed fracture over the centre of the superior longitudinal sinus but did not lose consciousness. Immediately after injury he developed slight weakness and numbness of both lower limbs, more marked on the left side than on the right, without disturbance of micturition or defaecation. At operation the depressed fragments were elevated and the dura shown to be intact. Subsequently this patient was left with slight residual spasticity in both lower limbs, again more in the left than the right. Encephalography showed bilateral general ventricular dilatation which was moderate on the right side and slight on the left.

These two cases indicate that ventricular dilatation can develop after head injuries in which consciousness was not lost. The responsible mechanism is not known. Possibly a thrombosis of the superior longitudinal sinus, directly resulting from the injury, may have played a part in the second case, but such a mechanism was unlikely in the first case.

\section{Head Injury without Residual Sequelæ}

Nine cases in the series eventually made complete clinical recoveries. In only one was the ventricular system normal. This case (Case 8 ) was the only example of a comparatively mild and straightforward head injury in our series. There was a post-traumatic amnesia of 2 hours. Encephalography was undertaken because, 1 hour after injury, a convulsive attack, the significance of which was not evident, had been observed down the right side of the body. The other 8 cases all showed ventricular enlargement. In one (Case 33 ) a symptomless focal dilatation of the left frontal horn developed beneath the site of a healed compound depressed fracture. The remaining 7 cases were all examples of prolonged post-traumatic confusion with confabulatory disturbances (Korsakow's psychosis). All seven made complete recoveries, and subsequently showed generalized dilatations of one or both lateral ventricles. It is interesting that two of them (Cases 61 and 88), both with well marked ventricular dilatation, were pilots who later returned to full flying duties.

It will be seen that, though this group includes only cases which made complete recoveries, it is really highly selected in that in most of the cases 
were examples of post-traumatic Korsakow's syndrome. It shows clearly that post-traumatic ventricular dilatation, even of moderate degree, is not necessarily associated with permanent psychological or neurological disturbances.

\section{Head Injury with Residual Neurological Signs}

\section{A. With Residual Pyramidal Signs}

Ventricular dilatation was observed in all except 2 of the 24 cases in this group. One of these two exceptions (Case 6) sustained a head injury of comparatively mild severity due to a blow on the right side of the head from a falling hammer. The skull was not fractured. The left arm was immediately paralysed, and slight permanent weakness of the arm was still evident 6 months later. The second exception (Case 9), was a case with a small, slightly depressed fracture in the mid-vertex over the superior longitudinal sinus, presenting slight residual sensory and motor signs in both lower limbs, more on the left side than on the right. A possible factor in the production of the clinical features shown by this case may have been a partial thrombosis of the superior longitudinal sinus beneath the depressed fracture.

In the remaining 22 cases ( 92 per cent.) the residual pyramidal disturbances were associated with ventricular dilatation. Six were examples of focal ventricular dilatation, 7 of unilateral ventricular dilatation, 6 of bilateral ventricular dilatation, and 3 of focal with unilateral general ventricular dilatation. It is interesting that with one exception the bigger ventricle was, appropriately, on the side opposite to that of the pyramidal disturbance. This relationship between the site of injury and the side on which symptoms appeared was further emphasized in Case 68, which showed bilateral pyramidal signs and bilateral ventricular dilatation; the side of greater ventricular dilatation was opposed to that of the greater motor signs. The exception to this rule was Case 44 , in which a pronounced residual left hemiparesis was observed in association with a marked focal dilatation of the anterior part of the left lateral ventricle; there may have been some dilatation of the vestibule of the right lateral ventricle in this case but the degree of enlargement was very slight and opinions varied as to whether it was of pathological degree.

Particularly striking were the 7 cases (Cases 51, $55,56,57,59,60,62)$ in which an apparently strictly unilateral ventricular dilatation was associated with corresponding pyramidal signs down the opposite side of the body. In one of these (Case 55) the degree of hemiparesis was very pronounced; in another (Case 57) the discrepancy in size between the two ventricles was particularly marked. An illustrative case history from this group is as follows:

Case 57.-A 24-year-old soldier received a closed head injury in April, 1940, as a result of a motor-cycle accident, which resulted in a post-traumatic amnesia of 2 weeks duration. Subsequently he complained of aphasia and of weakness of the right arm and leg which cleared up completely within a further 2 weeks. About 3 months after his injury he developed frequent epileptic attacks, which began with clonic movements of the fingers of the right hand, followed shortly by loss of consciousness for a few minutes. After many of the attacks he had aphasia and a transient right hemiplegia lasting for an hour. Between attacks weakness of the right lower face was evident, but no weakness or sensory disturbances in the limbs. X-ray examination showed a linear fracture in the left parietal region, and cisternal encephalography demonstrated a moderate dilatation of the whole of the left lateral ventricle. In view of the focal aura of his major attacks a left lateral craniotomy was performed and a small meningo-cerebral scar was found in the left pre-central gyrus.

This association of residual pyramidal signs with dilatation of the opposite ventricle occurs so frequently that it raises the question whether cases in which the pyramidal signs were only transient might not also show ventricular dilatation when examined at a later date. Our information as to the detailed signs shown by our cases in the acute stage is unfortunately incomplete, and we have records of only 7 such cases with transient pyramidal disturbances (Cases 58, 65, 86, 87, 89, 90, 100). It is interesting that either unilateral or bilateral ventricular dilatation was later demonstrated in all of them, and that the larger ventricle was always on the side opposite to that of motor dysfunction.

\section{B. With Hypothalamic and/or Brain Stem Signs}

Of the 4 cases included in this subgroup, only one (Case 41) showed ventricular dilatation. None had a fracture of the skull visible in the X-ray films. Three cases (Cases 28, 30, and 41), each with a prolonged post-traumatic amnesia of between 2 and 4 weeks duration, exhibited residual "cerebellar" ataxia down one side of the body without motor weakness. In one instance this was associated with nystagmus and dysarthria as well. The traumatic lesion responsible for these features was presumed to lie within either the brain stem of the cerebellum. Two of these three cases had normal encephalograms and the third some focal dilatation of the ipsolateral frontal horn. The remaining case (Case 22) in this subgroup was thought clinically to have had neurological lesions in the hypothalamus and upper part of the brain stem, which radiological examination showed were associated with a normal encephalogram. During the acute phase immediately following injury, the characteristic postures of decerebrate rigidity, associated with righting reflexes of the Magnus and de Kleyn type, were demonstrated. In the later stages the patient was left with a profound dementia, slight residual weakness of the right arm, and increased spasticity of both lower limbs. These four cases together suggest that cases of primary brain stem and hypothalamic injury are not usually followed by ventricular dilatation.

\section{With Residual Optic Radiation Defects}

An incomplete homonymous hemianopia of congrous type was observed in three cases (Cases 25, 49, 75). In each case there was a fracture in the parietooccipital region, presumably associated with a 
localized injury of the posterior part of the cerebral hemisphere. In no case did a dilatation of the related part of the lateral ventricle occur. In the first case (Case 25) the ventricular outlines were normal, in the second case (Case 49) there was a generalized dilatation of the opposite lateral ventricle from contrecoup, while in the third case (Case 75) there was a generalized symmetrical dilatation of both lateral ventricles. These three cases would seem to indicate that a localized cerebral injury is not necessarily followed by ventricular dilatation, but it must be remembered that ventricular changes in the occipital horns are often difficult to assess, owing to the wide range of normal anatomical variations.

\section{With Cranial Nerve Palsies}

In this group are included only cases in which cranial nerves were damaged, presumably within the cranial cavity, by injuries such as those involving the olfactory and oculomotor nerves or the optic chiasm. Cases in which the nerve was obviously damaged in its course through the skull, as in lesions of the optic nerve, the facial and auditory nerves, and the divisions of the trigeminal nerve were excluded.

Sixteen cases were found in the series, some with multiple lesions; 12 showed anosmia, 4 chismatic disturbances, and 3 oculomotor nerve palsies. The majority were associated with other residual neurological lesions which rendered it very difficult to determine the relationship between the state of the ventricles and the cranial nerve palsies. In 5 cases, however, cranial nerve palsies were noted as occurring without other residual sequelæ. Two of these cases showed bilateral anosmia and bitemporal hemianopia, one bilateral anosmia, one bilateral sixth nerve paresis, and one unilateral sixth nerve palsy. The ventricles were normal in three, showed focal dilatation in a fourth, and focal with generalized dilatation in the fifth. There is probably no direct relationship between the state of the ventricles and these cranial nerve palsies.

\section{Post-Traumatic Syndrome and Post-Traumatic In- tellectual Deterioration}

Residual symptoms of intellectual disturbances, either subjective or objective, were observed in 53 cases. According as to whether subjective or objective features predominated, we divided these cases, as mentioned earlier, into two groups, a group of cases of post-traumatic syndrome in which headaches, giddiness, and lassitude were the characteristic features, and a group of cases of post-traumatic intellectual deterioration in which objective personality changes were conspicuous.

Twenty-nine cases were listed as examples of the post-traumatic syndrome. Collectively they were the result of injuries which clinically seemed less severe than those in the second group. In 3 out of the 29 cases $(10$ per cent.) there had not been any loss of consciousness, in 16 cases ( 55 per cent.) the post-traumatic amnesia was of 1 week or less, while in only 10 cases ( 35 per cent.) was it greater than 1 week. Radiological examination showed that of the cases of post-traumatic syndrome nearly half (14 cases, i.e. 48 per cent. of the group) showed normal ventricular outlines. In addition 3 cases (10 per cent.) showed focal enlargement of the ventricles, 6 (21 per cent.) unilateral ventricular dilatation, and 4 (14 per cent.) bilateral ventricular dilatation, while 2 cases ( 7 per cent.) showed combinations of focal and generalized ventricular dilatation.

Twenty-four cases were included in the second group of post-traumatic intellectual deterioration. By comparison with the first group, the injuries in this group were clinically more severe and were followed more frequently by ventricular dilatation. Thus a post-traumatic amnesia of one week or less was observed in only 6 cases ( 25 per cent.), whereas in 18 cases ( 75 per cent. as opposed to 35 per cent. in cases of post-traumatic syndrome) the duration of the post-traumatic amnesia exceeded a week. No cases of head injury without loss of consciousness were included in this group. Twenty-one out of the 24 cases (88 per cent.) showed ventricular dilatation. In 50 per cent. of these cases ( 12 cases), both lateral ventricles were affected; unilateral ventricular dilatation was observed in 4 cases (17 per cent.); focal ventricular enlargement in 5 cases ( 21 per cent.), and normal ventricular outlines in 3 cases (12 per cent.).

\section{Post-Traumatic Epilepsy}

Recurrent epileptic phenomena considered to be the direct result of the head injury were noted in 19 cases. Cases of epilepsy with antecedent attacks or with a known family history of epilepsy were excluded, as were all cases in which convulsive disturbances occurred only during the acute phases following injury. Grand mal seizures were observed in 12 out of the 19 cases, petit mal attacks in 2, grand mal associated with petit mal in 3, and focal motor seizures in 2 cases. Usually these attacks appeared some months after the injury, but in one case (Case 47), they apparently first appeared within a few hours of the accident, while in another case (Case 5), an interval of 3 years elapsed before the attacks appeared.

The incidence of post-traumatic epilepsy in our series appeared to bear no relation to the severity of the responsible injury, as measured by either the duration of the post-traumatic amnesia or the degree of ventricular dilatation. Thus 3 cases sustained injuries without loss of consciousness, 3 had posttraumatic amnesia of less than 1 day, 6 post-traumatic amnesia of 1 week, and 7 post-traumatic amnesia of more than 1 week. Again, of the 10 cases in which post-traumatic epilepsy occurred without other residual features, half showed normal ventricles and half dilated ventricles.

\section{Discussion}

It is evident from this analysis of our case material that ventricular dilatation of either a focal or a generalized character often follows closed head 
injuries. Although dilatation may occur after apparently mild injuries, the frequency with which it is found increases with the severity of the trauma. These ventricular enlargements first appear within 2-3 weeks of injury and may reach their maximum within a month. They do not appear to be influenced so much by the site of the direction of the violence as by the presence of a fracture of the skull. Focal dilatations in particular tend to develop beneath the site of a localized fracture of the skull.

Although in some cases the correlation between the clinical signs and the encephalographic appearances is not at first apparent, in most there is an obvious relationship between the two. In particular, cases with residual pyramidal signs down one side of the body are frequently found to have dilatation of the lateral ventricle on the opposite side of the brain. Moreover, some of the remaining cases of ventricular enlargement were known to have presented signs of pyramidal disturbance in the acute phase of injury. Again, in cases with marked intellectual and mental deterioration both ventricles are often enlarged, whereas in cases without objective deterioration, but with only disorders of a subjective character such as headaches and dizziness, the ventricular system is more often normal. On the other hand the appearance of post-traumatic epilepsy does not seem to be connected with changes in the ventricular outline.

We strongly deprecate the view expressed by Swift (1932), Hauptmann (1931), Friedman (1932), and others that air encephalography can be used as a certain means of differentiating between cases with genuine disabilities and those with functional disturbances. These authorities, who have advocated the use of air encephalography in this connexion, have implied that, when the encephalograms are normal, the case should be regarded as of functional character. In our series of 31 cases with normal ventricular outlines following head injury, at least 6 cases had definite objective neurological signs indicating cerebral damage and 6 suffered from post-traumatic epilepsy. We therefore feel that the findings of a normal encephalogram in a given case does not prove that the symptoms are functional, because permanent organic damage can occur in the brain without resulting in later ventricular enlargement.

In this paper we have confined ourselves to a consideration of the ventricular changes, and have not paid attention to the other encephalographic features described in Bielschowsky's classical paper (1928). We do not consider non-filling of the ventricles with air to be evidence of pathological change, for we have observed on several occasions, after encephalography had failed to fill the ventricles, that ventriculography has revealed a normal ventricular system. Recently we met with a case in which, after lumbar encephalography had failed to outline the ventricles, encephalography repeated a few days later with air introduced by the cisternal route produced very satisfactory filling of the ventricles, which appeared normal. As during the period when our 100 cases were investigated there were only 4 cases of chronic head injury in which ventricular filling was not obtained, we have not felt justified in considering such cases as pathological and have consequently excluded them from our series.

Authors using lumbar encephalography have rightly laid great emphasis on abnormalities of distribution of the subarachnoid air. In our material these abnormalities have not been obvious, except in cases which also showed ventricular changes, and so we have not considered them. Cisternal encephalography, as performed in this hospital with the installation of only small quantities of air, does not lend itself to so clear a demonstration of the subarachnoid pathways as does lumbar encephalography. We feel this disadvantage of cisternal encephalography is outweighed by the advantage of being able to outline the ventricles clearly with smaller quantities of air, thereby causing much less distress to the patient.

The presence of ventricular dilatation after recovery from a head injury indicates that an atrophic process has taken place in the brain. Such an atrophic process may be the result of obstruction to the cerebro-spinal fluid pathways, or of resolution of the pathological changes produced in the brain itself by the trauma. Broadly speaking, posttraumatic ventricular dilatations are of two types, focal and generalized, and are probably produced by different mechanisms. It is unlikely that purely focal dilatations, for example, are due to the back pressure effects of obstruction to the cerebro-spinal fluid pathways, although such factors may operate in producing generalized dilatations. It is more likely that focal dilatation is the result of resolution of local pathological changes such as focal hæmorrhage, contusion, and laceration of brain tissue directly occasioned by the injury. The frequency with which focal dilatations develop beneath a localized fracture of the skull supports this view. In the production of generalized dilatation, other factors, which affect the brain more widely, must also operate. It is known that after head injury widespread pathological changes may occur in the brain, often remote from the actual site of injury, and may even overshadow the local injury (Hassin, 1940). Factors, such as odema of the cerebral hemispheres and dilatation of the cerebral capillaries and veins, often with endarteritis (Winkelman and Eckel, 1934), may play a part in the widespread pathological processes. Traumatic subarachnoid hæmorrhage may also contribute to a generalized bilateral ventricular dilatation, as indicated by the experimental observations of Bagley (1928). However, it is difficult to explain why ventricular dilatation is apparently so frequently confined to one lateral ventricle, and may even reach a considerable degree in that ventricle without the other lateral ventricle being affected. It is unlikely that traumatic subarachnoid hæmorrhage or obstruction to the cerebro-spinal pathways, which would affect both ventricles equally, here play a part. It seems more 
likely that the dilatation of the ventricle is the result of a vascular disturbance.

Whatever the explanation of the ventricular changes, the fact remains that, in addition to its use in demonstrating the presence of space-occupying lesions such as intra-cranial hæmatomata, air encephalography is a useful adjunct in the investigation of chronic head injuries. It affords a measurable indication of the extent of the gross atrophying processes consequent upon cerebral contusion, laceration, and traumatic vascular disturbance. It has its limitations in that finer degrees of cerebral injury can occur without subsequent alteration of the ventricular outlines.

\section{Summary}

1. The previous literature upon the subject of air encephalography after head injury shows that since 1928 there has been no review of any large series of cases from the aspect of correlation of the radiographic appearances with the clinical findings. In view of the improved clinical standards of assessment it was felt that the significance of the encephalographic changes should be evaluated in the light of these.

2. A series of 100 cases of closed head injury is presented, all of which were examined by a standardized technique, and a method of measurement of the ventricular bodies for the assessment of dilatation is described.

3. As control a series of 50 cases, in which the ventricular system was considered to be normal, was similarly examined.

4. The types of change in the ventricular outline encountered are described, and their time of onset and relation to the site of injury, to fracture and to the symptoms and physical signs presenting are discussed.

5. It would appear from the material that there is correlation between the ventricular changes and the clinical signs in the majority of cases, but that this may not always be so.

6. The mechanism of ventricular dilatation appears more likely to be due to intra-cerebral atrophic processes than to obstruction of the cerebro-spinal fluid pathways, as has previously been held by some writers.

7. Air encephalography cannot be regarded as a certain means of differentiating organic from functional post-traumatic states, as has been claimed. It does, however, afford a measurable criterion of the extent of atrophic processes occurring after cerebral damage.

We are much indebted to the medical officers of the military hospital for head injuries, under whose care these cases were, for their unfailing co-operation, and we wish to record our thanks to our Commanding Officer, Lieut.-Col. G. O. Chambers, M.C., for his permission to publish this paper. We also wish to express our thanks to T./Brigadier Hugh Cairns for his help and interest in this survey.

\section{REFERENCES}

Abramovitch, D., and Winkler H. (1930). Z. ges. Neurol. Psychiat., 127, 454.

Bagley, A. (1928). Arch. Surg., 17, 18.

Bennett, A. E., and Hunt, H. B. (1933). Arch. Surg., 26, 397.

Bielschowsky, P. (1928). Z. ges. Neurol. Psychiat., 117, 55.

Cairns, H. (1941). Brit. med. J., (ii), 465.

Davidoff, L. M., and Dyke, C. G. (1937). The Normal Encephalogram, Lea, Philadelphia.

Evans, W. A. (1942). Arch. Neurol. Psychiat. (Chicago), 47, 931 .

Fischer, M. (1927). Arch. Psychiat. Nervenkr., 82, 403.

Flugel, F. E. (1932). Ergebn. inn. Med. Kinderheilk., 44, 327.

Friedman, E. D. (1932). Arch. Neurol. Psychiat. (Chicago), 27, 791.

Hassin, G. B. (1940). Injuries of the Skull, Brain and Spinal Cord (Edited Brock), London.

Hauptmann, A. (1932). Arch. Psychiat. Nervenkr., 96, 84.

Lippens, A., and Desjardin, L. (1934). Pr. méd., 42, 455.

Lysholm, E. (1935). Acta radiol. Stockh., Supp. xxiv.

Money, R. A., and Susman, E. (1932). Aust. N. Z. J. Surg., $2,47$.

Schwab, O. (1926a). Z. ges. Neurol. Psychiat., 102, 294. (1926b). Dtsch. Z. Nervenheilk, 89, 44.

Swift, G. W. (1931). Surg. Gynec. Obstet., 52, 576.

Travers, J. T. (1937). Radiology, 28, 704.

Winkelman, N. W., and Eckel, J. L. (1934). Arch. Neurol Psychiat., 31, 956. 